
$\angle$ Research Square
Preprints are preliminary reports that have not undergone peer review.
They should not be considered conclusive, used to inform clinical practice, or referenced by the media as validated information.

\title{
Sensory Geneidentificationin the Transcriptome of the Ectoparasitoid Quadrastichus Mendeliinthe Eucalyptus Gall wasp Leptocybe Invasa
}

\author{
You Huang \\ Guangxi University \\ Yun Wang \\ Guangxi University \\ Wen Lu \\ Guangxi University \\ Lin Zheng ( $\square$ zheng-xia-lin@163.com ) \\ Guangxi University
}

\section{Research Article}

Keywords: Parasitoids, Gall-making pest, Host location, Transcriptome, Chemosensory gene, Phylogenetic analysis

Posted Date: December 11th, 2020

DOI: https://doi.org/10.21203/rs.3.rs-123482/v1

License: @ (i) This work is licensed under a Creative Commons Attribution 4.0 International License. Read Full License 


\section{Abstract}

Gall-inducing insects live within plant tissues and induce tumor-like growth that provides insects with food, shelter, and protection from natural enemies. Interestingly, these insects can be precisely targeted by parasitoids. However, the chemical mechanism of the host location by parasitoids of gall-inducing insects has not been elucidated. Empirical evidence has shown that sensory genes play a key role in the host location of parasitoids. To date, the sensory genes that regulate parasitoids to locate gall-inducing insects have not been uncovered. An obligate ectoparasitoid, Quadrastichus mendeli Kim \& La Salle (Hymenoptera: Eulophidae: Tetrastichinae), is one of the most important parasitoids of Leptocybe invasa, which is a global gall-making pest in eucalyptus plantations. Therefore, Q. mendeli - L. invasa provides an ideal system to study the way that parasitoids use sensory genes in gall-making pests. In this study, we present the transcriptome of $Q$. mendeli using high-throughput sequencing. In total, 31820 transcripts were obtained and assembled into 26925 unigenes in Q. mendeli. Then, the major sensory genes were identified, and phylogenetic analyses were performed with these genes from Q. mendeli and other model insect species. Three chemosensory proteins (CSPs), 10 gustatory receptors (GRs), 21 ionotropic receptors (IRs), 58 odorant binding proteins (OBPs), 30 odorant receptors (ORs) and 2 sensory neuron membrane proteins (SNMPs) were identified in Q. mendeli by bioinformatics analysis. Our report is the first to obtain abundant biological information on the transcriptome of $Q$. mendeli that provided valuable information regarding the molecular basis of $Q$. mendeli perception, and it may help to understand the host location of parasitoids of gall-making pests.

\section{Introduction}

Sensory genes play a key role in the life of parasitoids, such as foraging, oviposition site selection, and mating partners ${ }^{1}$. There are two major chemosensory mechanisms through olfaction and taste in which chemical signals are detected by one of the large multigene families that encode chemosensory proteins (CSPs), gustatory receptors (GRs), ionotropic receptors (IRs), odorant-binding proteins (OBPs), sensory receptors (ORs) and sensory neuron membrane proteins (SNMPs) $^{2}$. The function of CSPs and OBPs is the first step in the recognition of chemical stimuli from the outside environment ${ }^{3}$. Chemoreceptors (such as GRs, IRs and ORs) are involved in the recognition and identification of various chemical signals and environmental odors to modulate chemical perception 4 . SNMPs are involved in cell signal transduction ${ }^{4}$.

Some sensory genes of parasitoids in Hymenoptera have been identified, including Bethylidae, e.g., Sclerodermus sp. ${ }^{2}$; Braconidae, e.g., Cotesia vestalis Haliday $^{5}$, Cot. chilonis Matsumura ${ }^{6}$, Microplitis demolitor Wilkinson ${ }^{7}$, M. mediator Haliday ${ }^{8}$, Microcentrus cingulum Brischke $^{9}$, Aphidius gifuensis Ashmead ${ }^{10}$, Ap. ervi Haliday ${ }^{11}$ and Meteorus pulchricornis Wesmael ${ }^{12}$; Encyrtidae, e.g., Anastatus japonicus Ashmead ${ }^{13}$ and Aenasius bambawalei Hayat $^{14}$; Eupelmidae, e.g., Copidosoma floridanum Ashmead ${ }^{15}$; Ichneumonidae, e.g., Campoletis chlorideae Uchida ${ }^{1}$; Trichogrammatidae, e.g., Trichogramma dendrolimi Matsumura ${ }^{16}$ and Tric. japonicun Ashmead ${ }^{3}$; Eulophidae, e.g., Asecodes hispinarum Boucek and Chouioia cunea Yang ${ }^{4,17}$. Previous studies have revealed that the sensory genes of parasitoids are involved in searching and locating wood-boring pests and leaf-mining pests ${ }^{18,19}$. For example, Scleroderma sichuanensis Xiao can accurately find the location of their hidden host Monochamus alternatue Hope and then parasitize them. SsicOBP1 and SsicOBP2 are the basis for the behavior of the odor, which have shown a strong reaction with (-)-a-pinene, (+)- $\beta$-pinene, camphene, and (+)-3-carene ${ }^{20}$. However, the sensory genes of parasitoids used to locate gall-making pests have not yet been solved, which has aroused great interest. Understanding this information can provide potential molecular targets for research based on reverse chemical ecology.

Quadrastichus mendeli Kim \& La Salle (Hymenoptera: Eulophidae) is one of the most important larval ectoparasitoids of Leptocybe invasa (Hymenoptera: Eulophidae), which is a global gall-making pest in eucalyptus plantations ${ }^{21,22}$. Q. mendeli is a uniparental parasitoid, and no males have been found ${ }^{23,24}$. Female Q. mendeli prefers to parasitize young and mature larvae of $L$. invasa with a percentage of parasitism of $84.20 \pm 11.40^{23}$. Q. mendeli has now been successfully established and effectively controls L. invasa populations in fields in Australia, China, Cambodia, India, Israel, Italy, Kenya, Laos, South Africa, Thailand and Vietnam ${ }^{25-29}$. Previous studies revealed that sensory genes of parasitoids play important roles in locating hosts ${ }^{8}$. Therefore, $Q$. mendeli - $L$. invasa is an ideal system to study the way that parasitoids use sensory genes in gall-making pests.

In this study, we performed high-throughput sequencing of the transcriptome and identified members of the major sensory genes that are crucial for $Q$. mendeli to locate L. invasa. Comparative analysis of the sensory genes in $Q$. mendeli with those in other species was also examined, and it provided valuable information regarding the molecular basis of $Q$. mendeli perception.

\section{Materials And Methods}

\section{Insects.}

Branches of saplings damaged by L. invasa were collected from Guangxi University ( $\left.108^{\circ} 29^{\prime} \mathrm{E}, 22^{\circ} 85^{\prime} \mathrm{N}\right)$, Nanning City, Guangxi Zhuang Autonomous Region, in October 2018. Specimens were placed in a glass container filled with water to retain freshness and transferred to a sealed net cage (length $\times$ width $\times$ height $=40 \mathrm{~cm} \times 40 \mathrm{~cm} \times 80 \mathrm{~cm}$ ) with $70-80 \%$ relative humidity and a natural light photoperiod maintained at $27 \pm 1{ }^{\circ} \mathrm{C}$. The water in the glass container was replaced daily until the end of the emergence of $Q$. mendeli. The emerged $Q$. mendeli were collected daily using 50 -mL plastic tubes. One day later, the tubes were immediately placed into liquid nitrogen and stored at $-80^{\circ} \mathrm{C}$. Six groups of female $Q$. mendeli adults (a group of approximately twenty) were prepared for RNA extraction.

\section{RNA sequencing.}

A NanoPhotometer ${ }^{\circledR}$ spectrophotometer (Thermo Fisher Scientific, MA, USA) and the Nano6000 Assay Kit for the Agilent Bioanalyzer 2000 system (Agilent Technologies, CA, USA) were applied to check the purity and integrity of the total RNA, respectively. After total RNA extraction, magnetic beads with Oligo dT

Page 2/27 
(Thermo Fisher Scientific, Hampton, USA) were used to enrich mRNA, and then, a fragmentation buffer was added to make it a short fragment. The fragments were sequenced on an Illumina HiSeq4000 (Illumina, San Diego, CA, USA).

\section{Transcriptome data analysis.}

Reads obtained from the sequencing machines included dirty reads containing adapters or low-quality bases, which affected the subsequent assembly and analysis. De novo transcriptome assembly was carried out with the short read assembly program Trinity v3.0 ${ }^{30}$. Basic annotation of unigenes includes protein functional annotation, pathway annotation, $\mathrm{COG} / \mathrm{KOG}$ functional annotation and Gene Ontology (GO) annotation. To annotate the unigenes, we used the BLASTx program (http://www.ncbi.nlm.nih.gov/BLAST/) with an E-value threshold of 1e-5 for the NCBI nonredundant protein (Nr) database (http://www.ncbi.nlm.nih.gov), the Swiss-Prot protein database (http://www.expasy.ch/sprot), the Kyoto Encyclopedia of Genes and Genomes (KEGG) database (http://www.genome.jp/kegg), and the COG/KOG database (http://www.ncbi.nlm.nih.gov/COG). Protein functional annotations could then be obtained according to the best alignment results ${ }^{31}$.

\section{Sequence alignment and phylogenetic analysis.}

Transmembrane domains (TMDs) were predicted using TMHMM 2.0 (http://www.cbs.dtu.dk/services/TMHMM) ${ }^{32}$. The signal peptides were predicted using SignalP 4.1 (http://www.cbs.dtu.dk/services/SignalP/) ${ }^{33}$. Amino acid sequence alignment was performed using the ClustalW method implemented in Mega v7.0 $0^{34}$. The $Q$. mendeli CSP, GR, IR, OR, OBP and SNMP nucleotide sequences were used as queries (BLASTx) in the GenBank database, and sequences from different insect species (i.e., Apis mellifera Ligustica, Bombyx mori Silk, C. floridanum, D. melanogaster, M. mediator, N. vitripennis, Solenopsis invicta Buren, Tribolium castaneum Herbst and Tric. Pretiosum Riley) and their amino acids were retrieved in the GenBank database and used to construct a phylogenetic tree. A. mellifera, B. mori, D. melanogaster and Trib. castaneum are model insects; S. invicta is a kind of social insect; $C$. floridanum, M. mediator and Tric. pretiosum are parasitoids from Hymenoptera. Amino acid sequences were aligned using the Muscle method implemented in Mega v7.035. The resulting alignment was manually curated to remove gap-rich regions. Maximum-likelihood trees (for CSP, GR, IR, OR, OBP and SNMP) were constructed using IQ-TREE with the best-fitting substitution model ${ }^{36}$. Subsequently, trees were viewed and graphically edited in FigTree v1.4.3 $3^{37}$ and Adobe Illustrator CS6. Branch support was assessed using the bootstrap method based on 1000 replicates.

\section{Results}

\section{Transcriptome assembly and annotation.}

To obtain high-quality clean reads, raw reads with adapters, low quality, and an $\mathrm{N}$ content greater than $10 \%$ were removed. The number of clean reads in female adults of $Q$. mendeli ranged from 18733252 to 25259462 , and the sample GC content ranged from $45.06-53.01 \%$ (Table 1 ). At the same time, Q20 and Q30 ranged from $92.75-94.36 \%$ and $88.45-90.08 \%$ respectively (Table 1). In total, 31820 transcripts were obtained and assembled into 26925 unigenes (Additional file 1). A total of $42.10 \%$ of unigenes had a length greater than 2000 bp and an average length of 1369 bp (Table 2). The NR database (15543, $57.73 \%$ ) had the largest match. In general, the sequences had E-values between 0 and $1 \mathrm{E}-150$ (Table 3 ). The e-value is an indication of the degree of similarity between the initial sequence used for searches and the sequence retrieved, and the higher the score, the greater the degree of similarity between them. The transcripts of Q. mendeli were most similar to the sequences of Nasonia vitripennis Walker (26.82\%), followed by the sequences of Ceratosolen solmsi marchali Mayr (7.49\%), Cop. floridanum (3.77\%), Tric. pretiosum (2.92\%), and other species (44.27\%) (Additional file 2). SwissProt (11644, $43.25 \%)$ and KOG $(10924,40.57 \%)$ shared similar quantities; KO $(7524,27.94 \%)$ showed the least match (Table 3$)$.

Table 1

Sequencing summary of the Quadrastichus mendeli transcriptome.

\begin{tabular}{|lllllll|}
\hline Sample name & Raw reads & Clean reads & Clean data (Gb) & Q20(\%) & Q30(\%) & GC content \\
\hline Q. mendeli 1 & 24740386 & 21110044 & 3.71 & 94.03 & 89.45 & 45.79 \\
\hline Q. mendeli 2 & 22581498 & 19278188 & 3.39 & 94.13 & 89.65 & 45.22 \\
\hline Q. mendeli 3 & 29819798 & 25259462 & 4.47 & 93.85 & 89.11 & 45.06 \\
\hline Q. mendeli 4 & 25051518 & 18733252 & 3.76 & 92.75 & 88.45 & 53.01 \\
\hline Q. mendeli 5 & 26482834 & 22495242 & 3.97 & 93.94 & 89.31 & 45.26 \\
\hline Q. mendeli 6 & 26557838 & 22736816 & 3.98 & 94.36 & 90.08 & 45.18 \\
\hline
\end{tabular}


Table 2

Number and length of transcripts and unigenes.

\begin{tabular}{|llll|}
\hline Length range/bp & Contig & Transcript & Unigene \\
\hline $0-300$ & $442916(95.09 \%)$ & $7976(25.07 \%)$ & $5859(21.76 \%)$ \\
\hline $301-500$ & $5879(1.26 \%)$ & $6191(19.46 \%)$ & $4816(17.89 \%)$ \\
\hline $501-1000$ & $5696(1.22 \%)$ & $5739(18.04 \%)$ & $4915(18.25 \%)$ \\
\hline $1001-2000$ & $5660(1.22 \%)$ & $5694(17.89 \%)$ & $5315(19.74 \%)$ \\
\hline 2001 & $11297(2.43 \%)$ & $11914(37.44 \%)$ & $11335(42.10 \%)$ \\
\hline Total number & 465788 & 31820 & 26925 \\
\hline Total length/bp & 66378092 & 39430660 & 36871436 \\
\hline N50 length/bp & 1617 & 2356 & 2504 \\
\hline Mean length/bp & 143 & 1239 & 1369 \\
\hline
\end{tabular}

Table 3

Unigenes annotated in different databases

\begin{tabular}{|c|c|c|c|c|c|c|c|}
\hline & $\begin{array}{l}1 \mathrm{E}-20<\text { evalue }<= \\
1 \mathrm{E}-5\end{array}$ & $\begin{array}{l}1 \mathrm{E}-50<\text { evalue }<= \\
1 \mathrm{E}-20\end{array}$ & $\begin{array}{l}1 \mathrm{E}-100<\text { evalue }<= \\
1 \mathrm{E}-50\end{array}$ & $\begin{array}{l}1 \mathrm{E}-150<\text { evalue }<=1 \mathrm{E}- \\
100\end{array}$ & $\begin{array}{l}0<=\text { evalue }<=1 \mathrm{E}- \\
150\end{array}$ & $\begin{array}{l}\text { Total } \\
\text { NO. }\end{array}$ & $\begin{array}{l}\text { PCT } \\
(\%)\end{array}$ \\
\hline KO & 809 & 1006 & 1140 & 835 & 3734 & 7524 & 27.94 \\
\hline KOG & 2027 & 2342 & 2545 & 1540 & 2470 & 10924 & 40.57 \\
\hline NR & 2495 & 2828 & 2334 & 1683 & 6203 & 15543 & 57.73 \\
\hline Swissprot & 2407 & 2847 & 2690 & 1477 & 2223 & 11644 & 43.25 \\
\hline
\end{tabular}

Abbreviations: NR: NCBI non-redundant protein sequences; Swissprot: A manually annotated and reviewed protein sequence database; KO: KEGG Orthology; KOG: Clusters of Orthologous Groups of proteins; Total NO.: Total number of annotated unigenes; PCT (\%): percentage (\%).

In total, 14735 were annotated into 52 subcategories belonging to three main GO categories: a 'biological process', 'cellular component' and 'molecular function' (Fig. 1a). There were 22 subcategories in the 'biological process', 18 subcategories in the 'cellular component', and 12 subcategories in the 'molecular function'. The top ten subcategories were 'catalytic activity' (1580), 'metabolic process' (1552), 'binding' (1552), 'cellular process' (1539), 'single-organism process' (1312), 'cell' (932), 'cell part' (932), 'membrane' (639), 'biological regulation' (615) and 'organelle' (576) (Additional file 3). By KOG classifications, 4689 unigenes were classified functionally into 25 categories (Fig. 1b). The cluster of 'general fractional prediction only' was the largest group, which had 4855 unigenes. The 'signal transduction mechanisms' group was second with 3998 unigenes. The top 2 categories had $36.64 \%$ unigenes annotated to the KOG database (Additional file 4). In total, 5160 unigenes were functionally classified into 5 KEGG categories (Fig. 1c). They were 'cellular processes' (587 unigenes, $7.66 \%$ of the unigenes annotated to the KEGG database), 'environmental information processing' (739, 9.64\%), 'genetic information processing' (1612, 21.03\%), 'metabolism' (4504, 58.77\%) and 'organismal systems' (222, 2.90\%) (Additional file 4). Among the 31 subcategories, 'Global and Overview' (2123, $27.70 \%)$, 'translation' $(664,8.66 \%)$ and 'Signal transduction' $(599,7.82 \%)$ were the top 3 (Additional file 5).

\section{Identification of candidate chemosensory genes.}

In this study, 3 putative unigenes encoding CSPs were identified, named QM_comp07737, QM_comp08732 and QM_comp26540 (Additional file 6). The lengths of these unigenes were 509 bp, 608 bp and 280 bp, respectively (Additional file 7). Among these unigenes, QM_comp07737 and QM_comp26540 were incomplete due to a lack of a $5^{\prime}$ or $3^{\prime}$ terminus, and the molecular weights of the unigenes were $18 \mathrm{kDa}$ and $9 \mathrm{kDa}$, respectively (Additional file 8 ).

QM_comp08732 sequences were full-length putative CSP genes because they had complete ORFs and 4 cysteines, which are characteristic of typical insect CSPs. QM_comp08732 with a molecular weight of $17 \mathrm{kDa}$ had a signal peptide sequence of approximately 22 amino acids at the $\mathrm{N}$-terminus (Additional file 8). Through a homology search with known proteins, the results showed that $73 \%$ of QM_comp26540 was orthologs of the proteins in Tenebrio molitor L., and the orthologs of other CSP sequences were also above $60 \%$ (Additional file 8 ). A phylogenetic tree based on the maximum likelihood method was constructed used the 3 CSP sequences of $Q$. mendeli along with 67 CSP sequences from 6 other species (i.e., A. mellifera, B. mori, D. melanogaster, M. mediator, S. invicta and Trib. castaneum) (Fig. 2 and Additional file 9). The phylogenetic tree showed that QM_comp26540 shares a high homology and is closely clustered with MmedCSP1, which has been functionally characterized, and QM_comp07737 and QM_comp08732 did not branch clusters with any other insects; they may be specific CSPs of Q. mendeli (Fig. 2).

\section{Identification of candidate gustatory receptors.}

Ten candidate GR proteins were identified from the data sets (Additional file 6). Among these unigenes, QM_comp03300, QM_comp23544, QM_comp24536 and QM_comp26507 were incomplete due to the lack of a 5' or 3' terminus. The lengths of these unigenes were between $297 \mathrm{bp}$ and $505 \mathrm{bp}$, and the molecular weights of these unigenes were between $5 \mathrm{kDa}$ and $17 \mathrm{kDa}$ (Additional file 7). QM_comp00164, QM_comp03333, QM_comp11847, QM_comp15910, QM_comp22611 and QM_comp22814 sequences were full-length putative GR genes because they had complete ORFs. These unigenes had molecular

Page 4/27 
weights that ranged between $4 \mathrm{kDa}$ and $56 \mathrm{kDa}$ and had a signal peptide sequence that ranged between 15 and 41 amino acids at the $\mathrm{N}$-terminus (Additional file 8). Through a homology search with known proteins, the results showed that 79\% of QM_comp15910 were orthologs of the proteins in Trichomalopsis sarcophagae Gahan. A phylogenetic tree based on the maximum likelihood method was constructed used the $10 \mathrm{GR}$ sequences of $Q$. mendeli along with 191 GR sequences from 9 other species (i.e., A. mellifera, B. mori, C. floridanum, D. melanogaster, M. mediator, N. vitripennis, S. invict, Trib. castaneum and Tric pretiosum) (Fig. 3 and Additional file 9). The phylogenetic tree showed that the 10 GRs of Q. mendeli shared high homology and closely clustered with the proteins in B. mori (Fig. 3).

\section{Identification of candidate ionotropic receptors.}

Twenty-one candidate IR proteins were identified from the data sets (Additional file 6). Among these unigenes, 13 unigenes were incomplete due to the lack of a $5^{\prime}$ or $3^{\prime}$ terminus. The length of these unigenes was between $214 \mathrm{bp}$ and $1970 \mathrm{bp}$, and the molecular weights of these unigenes were between $6 \mathrm{kDa}$ and $17 \mathrm{kDa}$ (Additional file 7). Eight unigenes were full-length putative IR genes because they had complete ORFs. These unigenes had molecular weights that ranged between $5 \mathrm{kDa}$ and $12 \mathrm{kDa}$ (Additional file 8). Through a homology search with known proteins, the results showed that $100 \%$ of the IRs in $Q$. mendeli were orthologs of the proteins in Asbolus verrucosus LeConte, and the orthologs of other IR sequences were also above $37 \%$ (Additional file 8 ). A phylogenetic tree based on the maximum likelihood method was constructed used the 21 IR sequences of $Q$. mendeli along with 131 IR sequences from 9 other species (i.e., A. mellifera, B. mori, C. floridanum, D. melanogaster, M. mediator, N. vitripennis, S. invict, Trib. castaneum and Tric. pretiosum) (Fig. 4 and Additional file 9). The 21 IRs of Q. mendeli along with 131 IRs from 9 other species (i.e., A. mellifera, B. mori, C. floridanum, D. melanogaster, M. mediator, N. vitripennis, S. invict, Trib. castaneum and Tric. pretiosum) were chosen to construct a phylogenetic tree based on the amino acid sequences (Additional file 9). The phylogenetic tree showed that all candidate IR proteins were clustered with at least one Hymenoptera ortholog (Fig. 4).

\section{Identification of candidate odorant binding proteins.}

Fifty-six candidate OBP proteins were identified from the data sets (Table 4; Additional file 6). Among these unigenes, 10 unigenes were incomplete due to the lack of a $5^{\prime}$ or $3^{\prime}$ terminus. The length of these unigenes was between $223 \mathrm{bp}$ and $768 \mathrm{bp}$, and the molecular weights of these unigenes were between $5 \mathrm{kDa}$ and $15 \mathrm{kDa}$ (Additional file 7). Forty-six unigenes were full-length putative OBP genes because they had complete ORFs. These unigenes had molecular weights ranging between $10 \mathrm{kDa}$ and $17 \mathrm{kDa}$ and had a signal peptide sequence ranging between 16 and 23 amino acids at the $\mathrm{N}$-terminus (Additional file 8 ). Insect OBPs can be classified into classical OBPs (six-cysteine conserved signature) and Minus-C (missing C2 and C5) and Plus-C (carries an additional conserved cysteine located between C1 and C2 and after C6). QM_comp02388, QM_comp07285, QM_comp08846, QM_comp10855, QM_comp21133, QM_comp21238 and QM_comp24139 had four conserved cysteines, which were minus-COBPs. Other OBPs had six conserved cysteines, which are classic OBPs. Plus-COBPs were not found in hymenopteran species. Through a homology search with known proteins, the results showed that $88 \%$ of QM_comp19239 were orthologs of the proteins in N. vitripennis, and the orthologs of other OBP sequences were also above 42\% (Additional file 8). A phylogenetic tree based on the maximum likelihood method was constructed used the 56 OBP sequences of $Q$. mendeli along with 209 OBP sequences from 9 other species (i.e., A. mellifera, B. mori, C. floridanum, D. melanogaster, M. mediator, N. vitripennis, S. invict, Trib. castaneum and Tric. pretiosum) (Fig. 5 and Additional file 9). The phylogenetic tree showed that all candidate OBP proteins were clustered with at least one Hymenoptera ortholog (Fig. 5). 
Table 4

Detailed information on the OBP ungenes of Quadrastichus mendeli.

\begin{tabular}{|c|c|c|c|c|c|c|c|c|c|c|c|c|}
\hline \multirow[t]{2}{*}{ Unigene ID } & \multirow{2}{*}{$\begin{array}{l}\text { Unigene } \\
\text { Length } \\
\text { (bp) }\end{array}$} & \multirow{2}{*}{$\begin{array}{l}\text { ORF } \\
\text { Length } \\
\text { (aa) }\end{array}$} & \multirow{2}{*}{$\begin{array}{l}\text { Complete } \\
\text { ORF }\end{array}$} & \multirow{2}{*}{$\begin{array}{l}5^{\prime} \text { or } 3^{\prime} \\
\text { Terminus } \\
\text { Lost }\end{array}$} & \multirow{2}{*}{$\begin{array}{l}\text { Signal } \\
\text { Peptide }\end{array}$} & \multirow{2}{*}{$\begin{array}{l}\text { Signal } \\
\text { Peptide } \\
\text { (aa) }\end{array}$} & \multirow{2}{*}{$\begin{array}{l}\text { Cysteine } \\
\text { Number }\end{array}$} & \multirow{2}{*}{$\begin{array}{l}\text { FPKM } \\
\text { (Mean) }\end{array}$} & \multicolumn{4}{|c|}{ Homology search with known proteins } \\
\hline & & & & & & & & & $\begin{array}{l}\text { Identity } \\
(\%)\end{array}$ & $\begin{array}{l}\mathrm{E} \\
\text { value }\end{array}$ & Species & Protein I \\
\hline QM_comp01352 & 728 & 132 & YES & - & YES & 18 & 8 & 14.67 & 37 & $\begin{array}{l}2 \mathrm{e}- \\
20\end{array}$ & $\begin{array}{l}\text { C. } \\
\text { floridanum }\end{array}$ & XP_014: \\
\hline QM_comp02170 & 549 & 129 & YES & - & YES & 19 & 7 & 234.80 & 53 & $\begin{array}{l}2 \mathrm{e}- \\
21\end{array}$ & N. vitripennis & XP_0016 \\
\hline QM_comp02233 & 411 & 129 & NO & $3^{\prime}$ & YES & 21 & 8 & 0.32 & 34 & $\begin{array}{l}2 \mathrm{e}- \\
07\end{array}$ & $\begin{array}{l}\text { C. } \\
\text { floridanum }\end{array}$ & XP_014: \\
\hline QM_comp02388 & 1415 & 125 & YES & - & NO & - & 5 & 1.67 & 58 & $\begin{array}{l}1 \mathrm{e}^{-} \\
150\end{array}$ & $\begin{array}{l}\text { Habropoda } \\
\text { laboriosa }\end{array}$ & KOC598 \\
\hline QM_comp02394 & 438 & 130 & YES & - & YES & 19 & 8 & 17.91 & 43 & $\begin{array}{l}5 e- \\
27\end{array}$ & N. vitripennis & XP_001t \\
\hline QM_comp02616 & 679 & 157 & YES & - & NO & - & 9 & 203.66 & 68 & $\begin{array}{l}2 \mathrm{e}- \\
46\end{array}$ & N. vitripennis & XP_001t \\
\hline QM_comp02693 & 407 & 118 & NO & $3^{\prime}$ & YES & 19 & 7 & 5.73 & 52 & $\begin{array}{l}7 e- \\
26\end{array}$ & N. vitripennis & XP_0016 \\
\hline QM_comp03741 & 518 & 129 & YES & - & YES & 19 & 7 & 1.12 & 40 & $\begin{array}{l}6 \mathrm{e}- \\
18\end{array}$ & N. vitripennis & XP_0016 \\
\hline QM_comp04039 & 793 & 133 & YES & - & YES & 18 & 9 & 5.00 & 34 & $\begin{array}{l}1 e^{-} \\
08\end{array}$ & $\begin{array}{l}\text { C. } \\
\text { floridanum }\end{array}$ & XP_014: \\
\hline QM_comp04767 & 321 & 41 & NO & $3^{\prime}$ & NO & - & 0 & 0.58 & 48 & $\begin{array}{l}2 \mathrm{e}- \\
09\end{array}$ & $\begin{array}{l}\text { C. } \\
\text { floridanum }\end{array}$ & XP_014: \\
\hline QM_comp05191 & 915 & 113 & YES & - & YES & 20 & 10 & 10.24 & 41 & $\begin{array}{l}7 e- \\
12\end{array}$ & $\begin{array}{l}\text { C. } \\
\text { floridanum }\end{array}$ & XP_014: \\
\hline QM_comp05917 & 727 & 133 & YES & - & YES & 19 & 6 & 35.75 & 41 & $\begin{array}{l}5 \mathrm{e}- \\
14\end{array}$ & N. vitripennis & XP_0016 \\
\hline QM_comp06244 & 433 & 127 & NO & $3^{\prime}$ & YES & 17 & 8 & 8.89 & 63 & $\begin{array}{l}5 e- \\
45\end{array}$ & T. dendrolimi & ANG085 \\
\hline QM_comp06765 & 917 & 150 & YES & - & YES & 23 & 7 & 3.90 & 52 & $\begin{array}{l}2 \mathrm{e}- \\
41\end{array}$ & $\begin{array}{l}\text { Ceratosolen } \\
\text { solmsi } \\
\text { marchali }\end{array}$ & XP_011! \\
\hline QM_comp07285 & 729 & 92 & YES & - & NO & - & 4 & 1.40 & 86 & $\begin{array}{l}1 \mathrm{e}- \\
09\end{array}$ & $\begin{array}{l}\text { C. } \\
\text { floridanum }\end{array}$ & XP_014: \\
\hline QM_comp08027 & 538 & 140 & YES & - & YES & 19 & 8 & 113.56 & 49 & $\begin{array}{l}2 \mathrm{e}- \\
39\end{array}$ & N. vitripennis & XP_001t \\
\hline QM_comp08037 & 736 & 134 & YES & - & YES & 19 & 9 & 192.11 & 37 & $\begin{array}{l}9 \mathrm{e}- \\
24\end{array}$ & N. vitripennis & XP_001t \\
\hline QM_comp08573 & 1211 & 133 & YES & - & YES & 21 & 6 & 4.55 & 36 & $\begin{array}{l}2 \mathrm{e}- \\
06\end{array}$ & $\begin{array}{l}\text { C. } \\
\text { floridanum }\end{array}$ & XP_014: \\
\hline QM_comp08613 & 542 & 113 & YES & - & YES & 20 & 9 & 21.22 & 44 & $\begin{array}{l}2 \mathrm{e}- \\
12\end{array}$ & $\begin{array}{l}\text { C. } \\
\text { floridanum }\end{array}$ & XP_014: \\
\hline QM_comp08638 & 476 & 130 & YES & - & YES & 20 & 7 & 311.84 & 51 & $\begin{array}{l}2 e^{-} \\
25\end{array}$ & $\begin{array}{l}\text { C. } \\
\text { floridanum }\end{array}$ & XP_014: \\
\hline QM_comp08676 & 507 & 135 & YES & - & YES & 17 & 7 & 275.44 & 69 & $\begin{array}{l}6 \mathrm{e}- \\
63\end{array}$ & N. vitripennis & XP_001t \\
\hline QM_comp08846 & 492 & 125 & YES & - & YES & 20 & 7 & 62.90 & 38 & $\begin{array}{l}6 \mathrm{e}- \\
08\end{array}$ & $\begin{array}{l}\text { C. } \\
\text { floridanum }\end{array}$ & XP_014: \\
\hline QM_comp08899 & 580 & 128 & YES & - & YES & 18 & 7 & 43.75 & 35 & $\begin{array}{l}1 \mathrm{e}- \\
12\end{array}$ & N. vitripennis & XP_001t \\
\hline QM_comp08900 & 484 & 129 & YES & - & YES & 18 & 7 & 125.85 & 36 & $\begin{array}{l}2 \mathrm{e}- \\
13\end{array}$ & N. vitripennis & XP_001t \\
\hline QM_comp09338 & 510 & 131 & YES & - & YES & 19 & 11 & 59.68 & 42 & $\begin{array}{l}4 \mathrm{e}- \\
23\end{array}$ & N. vitripennis & XP_001t \\
\hline QM_comp09339 & 507 & 131 & YES & - & YES & 19 & 9 & 176.04 & 51 & $\begin{array}{l}3 e- \\
19\end{array}$ & N. vitripennis & XP_001t \\
\hline
\end{tabular}




\begin{tabular}{|c|c|c|c|c|c|c|c|c|c|c|c|c|}
\hline \multirow[t]{2}{*}{ Unigene ID } & \multirow{2}{*}{$\begin{array}{l}\text { Unigene } \\
\text { Length } \\
\text { (bp) }\end{array}$} & \multirow{2}{*}{$\begin{array}{l}\text { ORF } \\
\text { Length } \\
\text { (aa) }\end{array}$} & \multirow{2}{*}{$\begin{array}{l}\text { Complete } \\
\text { ORF }\end{array}$} & \multirow{2}{*}{$\begin{array}{l}5^{\prime} \text { or } 3^{\prime} \\
\text { Terminus } \\
\text { Lost }\end{array}$} & \multirow{2}{*}{$\begin{array}{l}\text { Signal } \\
\text { Peptide }\end{array}$} & \multirow{2}{*}{$\begin{array}{l}\text { Signal } \\
\text { Peptide } \\
\text { (aa) }\end{array}$} & \multirow{2}{*}{$\begin{array}{l}\text { Cysteine } \\
\text { Number }\end{array}$} & \multirow{2}{*}{$\begin{array}{l}\text { FPKM } \\
\text { (Mean) }\end{array}$} & \multicolumn{4}{|c|}{ Homology search with known proteins } \\
\hline & & & & & & & & & $\begin{array}{l}\text { Identity } \\
\text { (\%) }\end{array}$ & $\begin{array}{l}\mathrm{E} \\
\text { value }\end{array}$ & Species & Protein I \\
\hline QM_comp09356 & 647 & 145 & YES & - & YES & 22 & 6 & 4.84 & 53 & $\begin{array}{l}5 e- \\
51\end{array}$ & N. vitripennis & XP_001t \\
\hline QM_comp09551 & 688 & 108 & YES & - & YES & 20 & 9 & 9.27 & 43 & $\begin{array}{l}1 \mathrm{e}- \\
08\end{array}$ & $\begin{array}{l}\text { C. } \\
\text { floridanum }\end{array}$ & XP_014: \\
\hline QM_comp10209 & 523 & 137 & YES & - & YES & 17 & 7 & 36.04 & 45 & $\begin{array}{l}5 \mathrm{e}- \\
30\end{array}$ & N. vitripennis & XP_001€ \\
\hline QM_comp10426 & 498 & 124 & YES & - & YES & 19 & 9 & 7.66 & 33 & $\begin{array}{l}1 \mathrm{e}- \\
08\end{array}$ & N. vitripennis & XP_001t \\
\hline QM_comp10855 & 879 & 144 & YES & - & NO & - & 4 & 8.33 & 28 & $\begin{array}{l}7 e- \\
07\end{array}$ & N. vitripennis & XP_001t \\
\hline QM_comp11668 & 700 & 126 & YES & - & YES & 18 & 8 & 45.36 & 34 & $\begin{array}{l}7 \mathrm{e}- \\
12\end{array}$ & $\begin{array}{l}\text { C. } \\
\text { floridanum }\end{array}$ & XP_014: \\
\hline QM_comp12532 & 510 & 135 & YES & - & YES & 16 & 7 & 3.52 & 74 & $\begin{array}{l}6 \mathrm{e}- \\
57\end{array}$ & N. vitripennis & XP_001t \\
\hline QM_comp12533 & 669 & 135 & YES & - & YES & 16 & 7 & 21.25 & 74 & $\begin{array}{l}2 \mathrm{e}- \\
56\end{array}$ & N. vitripennis & XP_001t \\
\hline QM_comp14843 & 1465 & 125 & YES & - & YES & 19 & 8 & 144.20 & 44 & $\begin{array}{l}4 \mathrm{e}- \\
14\end{array}$ & $\begin{array}{l}\text { C. } \\
\text { floridanum }\end{array}$ & XP_014: \\
\hline QM_comp18897 & 568 & 132 & YES & - & YES & 20 & 9 & 2.74 & 26 & $\begin{array}{l}4 e_{-}^{-} \\
06\end{array}$ & N. vitripennis & XP_001t \\
\hline QM_comp20903 & 596 & 130 & YES & - & YES & 20 & 11 & 13.86 & 27 & $\begin{array}{l}1 \mathrm{e}- \\
05\end{array}$ & $\begin{array}{l}\text { C. } \\
\text { floridanum }\end{array}$ & XP_014: \\
\hline QM_comp21133 & 508 & 141 & YES & - & NO & - & 7 & 0.95 & 29 & $\begin{array}{l}3 e- \\
05\end{array}$ & $\begin{array}{l}\text { Ceratosolen } \\
\text { solmsi } \\
\text { marchali }\end{array}$ & XP_011! \\
\hline QM_comp21238 & 482 & 104 & YES & - & NO & - & 2 & 0.86 & 88 & $\begin{array}{l}1 \mathrm{e}- \\
43\end{array}$ & N. vitripennis & XP_016\{ \\
\hline QM_comp21371 & 321 & 100 & NO & $3^{\prime}$ & YES & 19 & 6 & 1.40 & 36 & $\begin{array}{l}3 e- \\
09\end{array}$ & N. vitripennis & XP_001t \\
\hline QM_comp21830 & 1294 & 144 & YES & - & YES & 23 & 9 & 73.07 & 79 & $\begin{array}{l}9 \mathrm{e}- \\
65\end{array}$ & N. vitripennis & XP_001t \\
\hline QM_comp21900 & 238 & 41 & NO & $3^{\prime}$ & NO & - & 1 & 0.67 & 51 & $\begin{array}{l}2 e- \\
16\end{array}$ & N. vitripennis & XP_001€ \\
\hline QM_comp23080 & 223 & 73 & NO & $3^{\prime}$ & YES & 19 & 5 & 0.59 & 32 & $\begin{array}{l}1 \mathrm{e}- \\
06\end{array}$ & N. vitripennis & XP_001t \\
\hline QM_comp23536 & 487 & 145 & YES & - & YES & 19 & 5 & 0.99 & 28 & $\begin{array}{l}6 \mathrm{e}- \\
07\end{array}$ & T. pretiosum & XP_014: \\
\hline QM_comp23819 & 378 & 120 & NO & $3^{\prime}$ & YES & 22 & 4 & 0.65 & 49 & $\begin{array}{l}4 e^{-} \\
29\end{array}$ & N. vitripennis & XP_001t \\
\hline QM_comp24139 & 1063 & 142 & YES & - & YES & 18 & 4 & 197.63 & 26 & $\begin{array}{l}3 e- \\
04\end{array}$ & $\begin{array}{l}\text { C. } \\
\text { floridanum }\end{array}$ & XP_014: \\
\hline QM_comp24881 & 456 & 124 & YES & - & YES & 17 & 8 & 3.80 & 26 & $\begin{array}{l}3 e- \\
04\end{array}$ & $\begin{array}{l}\text { C. } \\
\text { floridanum }\end{array}$ & XP_015t \\
\hline QM_comp24882 & 483 & 133 & YES & - & YES & 17 & 8 & 1.79 & 28 & $\begin{array}{l}2 \mathrm{e}- \\
04\end{array}$ & T. pretiosum & XP_014: \\
\hline QM_comp26560 & 572 & 145 & YES & - & YES & 22 & 6 & 40.28 & 69 & $\begin{array}{l}5 e- \\
66\end{array}$ & N. vitripennis & XP_016\{ \\
\hline QM_comp26834 & 545 & 126 & YES & - & YES & 17 & 8 & 249.66 & 73 & $\begin{array}{l}8 \mathrm{e}- \\
49\end{array}$ & $\begin{array}{l}\text { C. } \\
\text { floridanum }\end{array}$ & AHE409 \\
\hline QM_comp03957 & 562 & 132 & YES & - & YES & 20 & 7 & 12.82 & 46 & $\begin{array}{l}6 \mathrm{e}- \\
28\end{array}$ & $\begin{array}{l}\text { C. } \\
\text { floridanum }\end{array}$ & XP_014: \\
\hline QM_comp04156 & 497 & 139 & NO & 5 & YES & 20 & 9 & 93.54 & 30 & $\begin{array}{l}2 \mathrm{e}- \\
10\end{array}$ & N. vitripennis & XP_016 \\
\hline QM_comp06538 & 520 & 139 & YES & - & YES & 20 & 7 & 2.40 & 77 & $\begin{array}{l}8 \mathrm{e}- \\
67\end{array}$ & T. dendrolimi & ANG084 \\
\hline
\end{tabular}




\begin{tabular}{|c|c|c|c|c|c|c|c|c|c|c|c|c|}
\hline \multirow[t]{2}{*}{ Unigene ID } & \multirow{2}{*}{$\begin{array}{l}\text { Unigene } \\
\text { Length } \\
\text { (bp) }\end{array}$} & \multirow{2}{*}{$\begin{array}{l}\text { ORF } \\
\text { Length } \\
\text { (aa) }\end{array}$} & \multirow{2}{*}{$\begin{array}{l}\text { Complete } \\
\text { ORF }\end{array}$} & \multirow{2}{*}{$\begin{array}{l}5^{\prime} \text { or } 3^{\prime} \\
\text { Terminus } \\
\text { Lost }\end{array}$} & \multirow{2}{*}{$\begin{array}{l}\text { Signal } \\
\text { Peptide }\end{array}$} & \multirow{2}{*}{$\begin{array}{l}\text { Signal } \\
\text { Peptide } \\
\text { (aa) }\end{array}$} & \multirow{2}{*}{$\begin{array}{l}\text { Cysteine } \\
\text { Number }\end{array}$} & \multirow{2}{*}{$\begin{array}{l}\text { FPKM } \\
\text { (Mean) }\end{array}$} & \multicolumn{4}{|c|}{ Homology search with known proteins } \\
\hline & & & & & & & & & $\begin{array}{l}\text { Identity } \\
(\%)\end{array}$ & $\begin{array}{l}\mathrm{E} \\
\text { value }\end{array}$ & Species & Protein \\
\hline QM_comp06539 & 593 & 139 & YES & - & YES & 20 & 7 & 43.82 & 73 & $\begin{array}{l}1 \mathrm{e}- \\
52\end{array}$ & T. pretiosum & XP_014: \\
\hline QM_comp10678 & 1344 & 136 & YES & - & YES & 20 & 6 & 21.55 & 51 & $\begin{array}{l}4 \mathrm{e}- \\
41\end{array}$ & $\begin{array}{l}T . \\
\text { sarcophagae }\end{array}$ & OXU167 \\
\hline QM_comp18896 & 1057 & 154 & YES & - & YES & 20 & 9 & 5.18 & 41 & $\begin{array}{l}4 \mathrm{e}- \\
25\end{array}$ & $\begin{array}{l}\text { C. } \\
\text { floridanum }\end{array}$ & XP_014: \\
\hline
\end{tabular}

\section{Identification of candidate odorant receptors.}

Thirty candidate OR proteins were identified from the data sets (Additional file 6). Among these unigenes, 21 unigenes were incomplete due to the lack of a $5^{\prime}$ or $3^{\prime}$ terminus. The lengths of these unigenes were between $208 \mathrm{bp}$ and $936 \mathrm{bp}$, and the molecular weights of these unigenes were between $3 \mathrm{kDa}$ and $34 \mathrm{kDa}$ (Additional file 7). Nine unigenes were full-length putative OR genes because they had complete ORFs. These unigenes had molecular weights ranging between $4 \mathrm{kDa}$ and $53 \mathrm{kDa}$ and had a signal peptide sequence ranging between 16 and 23 amino acids at the $\mathrm{N}$-terminus (Additional file 8). Through a homology search with known proteins, the results showed that $95 \%$ of QM_comp14333 were orthologs of the proteins in C. cunea, and the orthologs of other OR sequences were also above $32 \%$ (Additional file 8). A phylogenetic tree based on the maximum likelihood method was constructed used the 30 oR sequences of $Q$. mendeli along with 235 OR sequences from 9 other species (i.e., A. mellifera, B. mori, C. floridanum, D. melanogaster, M. mediator, N. vitripennis, S. invict, Trib. castaneum and Tric. pretiosum) (Fig. 6 and Additional file 9). The phylogenetic tree showed that all candidate OR proteins were clustered with at least one Hymenoptera ortholog (Fig. 6).

\section{Identification of candidate sensory neuron membrane receptors.}

Two candidate SNMP proteins were identified from the data sets (Additional file 6). QM_comp21591 was incomplete due to the lack of a 3' terminus. The length of this unigene was $355 \mathrm{bp}$, and the molecular weight of this unigene was $14 \mathrm{kDa}$ (Additional file 7). QM_comp09081 was a full-length putative SNMP gene because it had complete ORFs. QM_comp09081 had a signal peptide sequence of 13 amino acids at the N-terminus, and the molecular weight of QM_comp09081 was 59 kDa (Additional file 8). According to sequence similarity, SNMP is divided into two SNMP subtypes, SNMP1 and SNMP2. Through a homology search with known proteins, the results showed that $67 \%$ of QM_comp09081 were orthologs of the proteins in N. vitripennis (Additional file 8). A phylogenetic tree based on the maximum likelihood method was constructed used the 2 SNMP sequences along with 25 SNMP sequences from 9 other species (i.e., A. mellifera, B. mori, C. floridanum, D. melanogaster, M. mediator, N. vitripennis, S. invict, Trib. castaneum and Tric. pretiosum) (Fig. 7 and Additional file 9). The phylogenetic tree showed that QM_comp21591 fell into the same clade as the insect SNMP1 group, and QM_comp09081 fell into the same clade as the insect SNMP2 group (Fig. 7).

\section{Discussion}

In this study, the major sensory genes (i.e., CSPs, GRs, IRs, OBPs, ORs and SNMPs), which perhaps regulate $Q$. mendeli to locate its host $L$. invasa, are first reported, providing valuable information for exploring how parasitoids use sensory genes to locate gall-making pests.

CSPs are widespread in the antenna and other chemical sensory organs of insects ${ }^{38}$ and are involved in the chemical perception and related behavior of insects $^{39}$. Compared to the total number of insects in the world, CSPs have been identified in only a few species of insects to different degrees, such as Coleoptera ${ }^{40}$, Hemiptera ${ }^{41}$, Hymenoptera ${ }^{12}$, Lepidoptera ${ }^{42}$ and Orthoptera ${ }^{43}$, whose numbers show interspecific diversity. For example, the number of CSPs varies from 4 CSPs in Drosophila melanogaster to 22 in B. mori ${ }^{44}$. In this study, 3 candidate CSPs were identified, which are less than those in the parasitoids Ch. cunea (11), Sclerodermus sp. (10), Me. pulchricornis (8) and Tric. dendrolimi (7) 2,4,12,16 and are greater than those in the parasitoids Cot. chilonis (2) and Ap. ervi $(2)^{6,11}$. Previous research confirmed that generalists seem to be specifically suited for the processing of odorant mixtures, and they respond in a similar manner to plant volatiles ${ }^{45}$. For example, Ch. cunea is a generalist and has multiple hosts (e.g., Stilpnotia salicis L., Ivela ochropoda Eversmann, Clostera anachoreta Fabricius, Semiothisa cinerearia Bremer \& Gray and Clania variegeta Snellen), while Cot. chilonis mainly parasitizes larvae of the genus Chilo Zincken ${ }^{46,47}$. It can be deduced that the number of CSPs relates to their host range. For the specialist $Q$. mendeli, the use of CSPs is expected to cope with the variability in host availability ${ }^{44}$. Previous studies also revealed that CSPs could be involved in the solubilization of hydrocarbons in the stratum corneum to recognize its companion ${ }^{48}$. Therefore, CSPs in Q. mendeli should be associated with its obligate parasitic characteristics, indicating that QmenCSPs may function in the chemical sensing of $L$. invasa and its shelter host eucalyptus trees. A similar story has been confirmed for $M$. mediator, which can accurately find and then parasitize its hidden host Agrotis segetum Denis and Schiffermüller ${ }^{49}$. Interestingly, MmedCSP1 had a strong reaction with methyl salicylate, pentane, ocimene, $\beta$-ionone, 3,4-dimethylbenzaldehyde, 2-hexanone and cis-3-hexe-1-ol, suggesting that MmedCSP1 can function in chemical sensing of the plant volatiles of $M$. mediator's host $A$. segetum ${ }^{49}$. Our results showed that QM_comp26540 is in the same clade as MmedCSP1, meaning that QM_comp26540 has the closest relationship with MmedCSP1. Encouragingly, significant GC-EAD responses of Q. mendeli antenna to 
eucalyptus volatile a-phellandrene and 1,8-cineole were observed ${ }^{50}$. Thus, QM_comp26540 may function as a chemosensor, which is involved in the process of recognizing plant volatiles from eucalyptus when $Q$. mendeli searches its host, $L$. invasa.

GRs are widespread in gustatory organs of insects that respond to various taste-related soluble compounds, and cuticular hydrocarbons and odorants, such as sugars, amino acids, salts, bitter compounds, $\mathrm{CO}_{2}$ and pheromones, can be recognized and combined by GRs ${ }^{51,52}$. To date, GRs in some model insects with genome reports have been identified, such as $A$. gambiae (76), B. mori (69), D. melanogaster (68) and N. vitripennis (58) $)^{53,54}$. In this study, 10 candidate GRs were identified, which was similar to other parasitoids, such as An. japonicus $(8)^{13}$, Sclerodermus sp. $(6)^{2}$ and $M$. mediator $(6)^{8}$. This could be attributed to the sequencing depth and species-functional specificity of GRs ${ }^{51}$. DmelGR5 and DmelGR64 in D. melanogaster are receptor proteins for sweet taste and are used to detect glucose, sucrose, maltose, maltitol and cottonseed sugar ${ }^{55}$. For $Q$. mendeli, females that were fed a honey solution or honey solution + young eucalyptus leaves lived for a longer time than those who underwent other treatments, including flowers, gall leaves, water, galled leaves + honey solution, no food and young leaves ${ }^{23}$. Therefore, GRs in Q. mendeli should play a key role in recognizing sugar and fresh eucalyptus leaves via various soluble compounds ${ }^{56}$. Interestingly, QM_comp11847 was in the same clade as the sugar receptor NvitGR1, which was used to recognize the only source of nutrients from host Lucilia caesar L. for the offspring of $N$. vitripennis ${ }^{57,58}$. Thus, QM_comp11847 may be involved in recognizing host organisms and sugars, which helps $Q$. mendeli to quickly access energy from these molecules.

IRs, which evolve from the ionotropic glutamate receptor (iGluR), are a new class of sensory proteins mainly in taste organs/sensilla that respond to food components, such as sugars, salts, water and bitter compounds, and detect small temperature differences ${ }^{59-61}$. In this study, 21 candidate IRs were identified, which was more than in the parasitoids Me. pulchricornis $(19)^{12}$, Ch. cunea $(10)^{4}$, M. mediator $(6)^{8}$, Sclerodermus sp. $(3)^{2}$, Mi. cingulum (3) ${ }^{9}$ and An. japonicus $(3)^{13}$. Physiological recordings from taste sensilla in $D$. Melanogaster and other insects have revealed responses of taste neurons to salts, sugars, water, bitter compounds and a large diversity of other tastants ${ }^{59,60}$. Taste sensilla are widely distributed on the antennae of $Q$. mendeli ${ }^{28}$, suggesting that QmenIRs may function as taste receptors. The phylogenetic tree showed that QmenIRs were spread across the tree branches and clustered with homologous IRs from other species, which suggested that QmenIRs may be functionally conserved. QM_comp21031 was located in the same clade as BmorIR21a of B. mori, DmelIR21a of D. melanogaster and TcasIR21a of Trib. castaneum, indicating that QM_comp21031 has the closest relationship with insect IR21a, which can mediate cool sensing in Drosophila ${ }^{62}$. Thus, QM_comp21031 may perceive changes in temperature since insect IR21a can achieve both heat avoidance and heating ${ }^{63,64}$. Taking the oviposition features into consideration, we deduced that female $Q$. mendeli may be capable of sensing surface heat on galls related to $L$. invasa damage, which requires further exploration.

OBPs are crucial in insect olfactory perception and are the first step in the recognition of chemical stimuli from the outside environment ${ }^{3}$. In some model insects, OBPs have been identified to different degrees, such as B. mori $(57)^{65}$, D. melanogaster $(51)^{66}$, Trib. castaneum (46) ${ }^{67}$ and A. gambiae $(44)^{68}$. In this study, 56 candidate OBPs were identified, which was more than in the parasitoids Ae. bambawalei $(54)^{14}$, Ch. cunea $(25)^{4}$, Tric. dendrolimi $(24)^{16}$, M. mediator $^{1}$ $(20)^{8}$, Me. pulchricornis $(16)^{12}$, T. japonicum $(15)^{3}$, Ap. ervi $(15)^{11}$, Sclerodermus sp. $(10)^{2}$, Cop. floridanum $(8)^{15}$, Cot. chilonis $(8)^{6}$ and As. hispinarum $(8)^{17}$. The number of $Q$. mendeli OBPs identified was less than that in the parasitoid Cot. vestalis $(74)^{5}$. Previous studies revealed that OBPs in parasitoids play a key role in binding and transporting hydrophobic odorants from the environment to sensory receptors ${ }^{69}$. In $Q$. mendeli, a significant behavioral response to the gall volatiles D-limonene and decanal was observed (unpublished data). Relevant QmenOBPs function in chemical sensing of these volatiles characterizing $L$. invasa and its shelter host eucalyptus trees, which bioinformatics analysis could help to target. Our results showed that QM_comp21238 is the same clade as MmedOBP10 of $M$. mediator, which is involved in the process of recognizing $\beta$-ionone and Nonanal when they find the location of their hidden host $A$. segetu $^{49,70}$. Thus, QM_comp21238 may also be involved in the process of recognizing similar odorants or ligands when $Q$. mendeli locates its shelter host, $L$. invasa.

ORs are thought to play critical roles in the perception of chemosensory stimuli by insects ${ }^{52}$. The number of ORs in parasitoids vary greatly ${ }^{1,4}$. In this study, 30 candidate ORs were identified, which was more than in the parasitoid Tric. dendrolimi $(9)^{16}$, Mi. cingulum $(9)^{9}$ and Sclerodermus sp. (8) ${ }^{2}$. Previous studies revealed that the $\mathrm{OR}$ of $M$. mediator play an important role in recognizing plant volatiles, such as nonanal and farnesene, which provided a key start to manipulate and develop ORs in wasps to find hosts and use them as biological tools for pest control ${ }^{71}$. The RNAi investigation of the role of MmedOrco, the M. mediator ortholog of Drosophila Or83b, supported the assumption that this highly conserved gene plays a similar role in insects ${ }^{71,72}$. QmenORs may function as chemoreceptors to recognize plant volatiles from eucalyptus. Our results showed that QM_comp20892 is in the same clade as DmelOR10a of $D$. melanogaster, which plays a role in responding to odorants such as methylsalicylate and acetophenone ${ }^{73,74}$. For $Q$. mendeli, QM_comp20892 may be involved in the process of recognizing similar odorants or ligands.

SNMPs are involved in cellular signal transduction and play a role in odor detection ${ }^{5}$. Two SNMPs are normally broadly identified in different insects, e.g., parasitoids Ch. cunea and Sclerodermus sp. ${ }^{2,4}$. It has been reported that SNMP1 and SNMP2 are both expressed in antennae sensilla and have different expression patterns ${ }^{4,75}$. In Ch. cunea, CcunSNMP1 is a morphine receptor of neurons, and CcunSNMP2 is mainly expressed in supporting cells and the lymph of antennal sensilla ${ }^{4}$, while the location and expression patterns of SNMPs in Q. mendeli should be further studied since this information should be associated with their functions. SNMP1 of $D$. melanogaster is involved in pheromone detection and enhances the $\mathrm{Ca}^{2+}$ responses served in signal transduction ${ }^{76}$. SNMP1 of M. mediator was determined to participate in both pheromone and general odor detection ${ }^{77}$. In contrast, the general functional mechanism of SNMP2 in parasitoids is still poorly understood. In addition, Q. mendeli is a uniparental parasitoid that is not required in the male search for mating ${ }^{22}$. Thus, the function of QmenSNMPs may include an oviposition pheromone receptor rather than a sex receptor and a membrane protein with unknown functions, which needs to be further explored. 
Chemical detection involves a series of complicated processes that require participation and interactions by multiple cascades of sensory proteins. Insect sensory proteins are capable of functional cooperation and division. First, OBPs and CSPs are both chemically binding proteins to various odorants and can also respond to the same chemicals, e.g., MmedOBP10 and MmedCSP1 are involved in the process of recognizing $\beta$-ionone and nonanal when they find the location of their hidden host $A$. segetu ${ }^{49,70}$. Second, ORs and IRs are both chemoreceptors, while there are differences in the process of recognizing odor substances $^{78}$. For example, IRs are better at detecting long-lasting odor pulses, and they are less sensitive, suggesting that they are better at close-range odor detection. In contrast, ORs are more sensitive and better at resolving brief (low molecular flux) pulsed stimuli ${ }^{78,79}$. Moreover, features of functional organization have emerged between behavioral response profiles of OBPs and electrophysiological response profiles of ORs ${ }^{73}$. Therefore, the sensory genes in Q. mendeli should systematically act on the process of locating their gall-making host, and the biological functions of these genes and their products are still poorly known. Overall the overall sensory genes of the wasp reported here provide valuable insight into the molecular mechanisms of olfaction, which help pave the way for the host location of $Q$. mendeli in gall-making pests.

\section{Declarations}

\section{Acknowledgments}

We thank Guang-Nan Zheng for his help with the insect rearing and sampling. This work was funded by National Natural Science Foundation of China (Grant No. 31870634), Guangxi Natural Science Foundation (grant No. 2018GXNSFAA138099), and Guangxi Scholarship Fund of Guangxi Education Department.

\section{Authors' contributions}

This experiment was conceived and coordinated by Z.Y.H., X.Y.W., W.L. and X.L.Z. in this study. Sampling was performed by Z.Y.H. and X.L.Z. Analysis of transcriptome data and sequence was performed by Z.Y.H. and X.Y.W. Z.Y.H., X.Y.W. and X.L.Z. drafted the manuscript. All authors read and approved the final version of the manuscript.

\section{Competing interests}

The authors declare that they have no competing interests

\section{References}

1. Sun, Y. et al. An odorant receptor mediates the attractiveness of cis-jasmone to Campoletis chlorideae, the endoparasitoid of Helicoverpa armigera. Insect Mol. Biol. 28, 23-24 (2018).

2. Zhou, C. X., Min, S. F, Yan, L. T. \& Wang, M. Q. Analysis of antennal transcriptome and odorant binding protein expression profiles of the recently identified parasitoid wasp, Sclerodermus sp. Comp. Biochem. Phys. D. 16, 10-19 (2015a).

3. Wu, J. D., Shen, Z. C., Hua, H. Q., Zhang, F. \& Li, Y. X. Identification and sex expression profiling of odorant-binding protein genes in Trichogramma japonicum (Hymenoptera: Trichogrammatidae) using RNA-Seq. Appl. Entomol. Zool. 52, 623-633 (2017).

4. Zhao, Y. et al. Transcriptome and expression patterns of chemosensory genes in antennae of the parasitoid wasp Chouioia cunea. Plos One 11, e0148159 (2016).

5. Nichols, Z. \& Vogt, R. G. The SNMP/CD36 gene family in Diptera, Hymenoptera and Coleoptera: Drosophila melanogaster, D. pseudoobscura, Anopheles gambiae, Aedes aegypti, Apis mellifera, and Tribolium castaneum. Insect Biochem. Molec. Biol. 38, 398-415 (2008).

6. Qi, Y. et al. Transcriptome analysis of an endoparasitoid wasp Cotesia chilonis (Hymenoptera: Braconidae) reveals genes involved in successful parasitism. Arch. Insect Biochem. 88, 203-221 (2014).

7. Zhou, X. et al. Chemoreceptor evolution in Hymenoptera and its implications for the evolution of eusociality. Genome Biol. Evol. 7, 2407-2416 (2015b).

8. Wang, S. N. et al. Characterization of antennal chemosensilla and associated odorant binding as well as chemosensory proteins in the parasitoid wasp Microplitis mediator (Hymenoptera: Braconidae). Sci. Rep. 8, 1-11 (2018).

9. Ahmed, T., Zhang, T., Wang, Z., He, K. \& Bai, S. Gene set of chemosensory receptors in the polyembryonic endoparasitoid Macrocentrus cingulum. Sci. Rep. 6, 7649 (2016).

10. Kang, Z. W. et al. Identification and expression analysis of chemosensory receptor genes in an aphid endoparasitoid Aphidius gifuensis. Sci. Rep. 7, 3939 (2017)

11. Ballesteros, G. I. et al. Expression differences in Aphidius ervi (Hymenoptera: Braconidae) females reared on different aphid host species. PeerJ 5, e3640 (2017).

12. Sheng, S. et al. Candidate chemosensory genes identified in the endoparasitoid Meteorus pulchricornis (Hymenoptera: Braconidae) by antennal transcriptome analysis. Comp. Biochem. Phys. D. 22, 20-31 (2017).

13. Li, H. et al. cDNA cloning, sequence analysis and expression profile of a chemosensory protein from the Clostera restitura (Lepidoptera: Notodontidae). Sci. Silvae Sin. 54, 67-75 (2018a). (in Chinese with English abstract)

14. Nie, X. P. et al. Antennal transcriptome and odorant binding protein expression profiles of an invasive mealybug and its parasitoid. J. Appl. Entomol. 142, 149-161 (2018). 
15. Donnell, D. M. Analysis of odorant-binding protein gene family members in the polyembryonic wasp, Copidosoma floridanum: Evidence for caste bias and host interaction. J. Insect Physiol. 60, 127-135 (2014).

16. Zhang, S. F. et al. Sensory and immune genes identification and analysis in a widely used parasitoid wasp Trichogramma dendrolimi (Hymenoptera: Trichogrammatidae). Insect Sci. 23, 417-429 (2016).

17. Li, K. et al. Identification of putative odorant binding protein genes in Asecodes hispinarum, a parasitoid of coconut leaf beetle (Brontispa longissima) by antennal RNA-Seq analysis. Biochem. Bioph. Res. Co. 467, 514-520 (2015a).

18. Sullivan, B. T., Pettersson, E. M., Seltmann, K. C. \& Berisford, C. W. Attraction of the bark beetle parasitoid Roptrocerus xylophagorum (Hymenoptera: Pteromalidae) to host-associated olfactory cues. Environ. Entomol. 29, 1138-1151 (2000).

19. Chen, H. Y. Studies on the role of sensilla ang mines in host location in Diglyphus isaea. Master' thesis, Chin Acad. Agri. Sci., Beijing, China. (2009) (in Chinese with English abstract)

20. Zhu, X. Q. Binding characterization of OBP1 and OBP2 in the Scleroderma sichuanensis Xiao and behavior verification. Master' thesis, Sichuan Agricultural University. Sichuan, China. (2017). (in Chinese with English abstract)

21. Mendel, Z., Protasov, A., Fisher, N. \& La Salle, J. Taxonomy and biology of Leptocybe invasa gen. \& sp. n. (Hymenoptera: Eulophidae), an invasive gall inducer on eucalyptus. Aust. J. Entomol. 43, 101-113 (2004).

22. Huang, Z. Y., Li, J., Lu, W., Zheng, X. L. \& Yang, Z. D. Parasitoids of the eucalyptus gall wasp Leptocybe invasa: a global review. Environ. Sci. Pollut. Res. 25, 29983-29995 (2018b).

23. Kim, I. K., Mendel, Z., Protasov, A., Blumberget, D. \& La Salle, J. Taxonomy, biology, and efficacy of two Australian parasitoids of the eucalyptus gall wasp, Leptocybe invasa Fisher \& La Salle (Hymenoptera: Eulophidae: Tetrastichinae). Zootaxa 1910, 1-20 (2008).

24. Zheng, X. L. et al. Parasitoids of the eucalyptus gall wasp Leptocybe invasa (Hymenoptera: Eulophidae) in China. Parasite 23, 58 (2016).

25. Dittrich-Schröder, G. et al. Biology and host preference of Selitrichodes neseri: a potential biological control agent of the eucalyptus gall wasp, Leptocybe invasa. Biol. Control. 78, 33-41 (2014).

26. Mendel, Z., Protasov, A. \& La Salle, J. Classical biological control of two eucalyptus gall wasps main outcome and conclusions. Biol. Control. 105, 66-78 (2017).

27. Bush, S. J. et al. First record of Quadrastichus mendeli a parasitoid of Leptocybe invasa in South Africa. South Forests 80, 275-277 (2018).

28. Huang, Z. Y. et al. Ultrastructure of female antennal sensilla of an endoparasitoid wasp, Quadrastichus mendeli Kim \& La Salle (Hymenoptera: Eulophidae: Tetrastichinae). Microsc. Microanal. 24, 431-441 (2018a).

29. Lawson, S. et al. Biological control of galling pests in eucalypt plantations in the Mekong region. Preprint at http://aciargovau/project/fst/2012/091 (2014).

30. Grabherr, M. G. et al. Full-length transcriptome assembly from RNA-Seq data without a reference genome. Nat. Biotechnol. 29, 644-652 (2011).

31. Conesa, A. et al. Blast2GO: a universal tool for annotation, visualization and analysis in functional genomics research. Bioinformatics 21, 3674-3676 (2005)

32. Krogh, A., Larsson, B., von Heijne, G. \& Sonnhammer, E. L. Predicting transmembrane protein topology with a hidden Markov model: application to complete genomes. J. Molec. Biol. 305, 567-580 (2001).

33. Petersen, T. N., Brunak, S., von Heijne, G. \& Nielsen, H. SignalP 4.0: discriminating signal peptides from transmembrane regions. Nat. Methods. 8, 785-786 (2011).

34. Thompson, J. D., Gibson, T. J. \& Higgins, D. G. Multiple sequence alignment using ClustalW and ClustalX. Current protocols in bioinformatics/editoral board, Andreas D. Baxevanis ... [et al.] Chapter 2, Unit 2, 3 (2002).

35. Kumar, S., Stecher, G. \& Tamura, K. MEGA7: Molecular evolutionary genetics analysis version 7.0 for bigger datasets. Mol. Biol. Evol. 33, 1870-1874 (2016).

36. Trifinopoulos, J., Nguyen, L.T., Von, H. A. \& Minh, B. Q. W-IQ-TREE: a fast online phylogenetic tool for maximum likelihood analysis. Nucleic Acids Res. 44, 232-235 (2016).

37. Wu, Z. et al. Differential expression analysis of chemoreception genes in the striped flea beetle Phyllotreta striolata using a transcriptomic approach. Plos One 11, e0153067 (2016).

38. Picimbon, J. F., Dietrich, K., Breer, H. \& Krieger J. Chemosensory proteins of Locusta migratoria (Orthoptera: Acrididae). Insect Biochem. Molec. 30, 233-241 (2000).

39. Sánchez-Gracia, A., Vieira, F. G. \& Rozas, J. Molecular evolution of the major chemosensory gene families in insects. Heredity 103, 208-216 (2009).

40. Li, X. M. et al. Candidate chemosensory genes identified in Colaphellus bowringi by antennal transcriptome analysis. BMC Genomics 16 (2009).

41. Song, Y. Q. et al. Analysis of the antennal transcriptome and chemoreception-related genes of the bean bug, Riptortus pedestris (Hemiptera: Alydidae). Acta Entomol. Sin. 60, 1120-1128 (2009). (in Chinese with English abstract)

42. Wang, X. Y., Xiong, M., Lei, C. L. \& Zhu, F. The developmental transcriptome of the synanthropic fly Chrysomya megacephala and insights into olfactory proteins. BMC Genomics 16, 20 (2015).

43. Zhou, Y. T. Identification and function analysis of olfactory proteins in Oedaleus asiaticus (Orthoptera: Acrididae). Master' thesis, Inner Mongolia University. Neimenggu, China. (2019). (in Chinese with English abstract)

44. Vieira, F. G. \& Rozas, J. Comparative genomics of the odorant-binding and chemosensory protein gene families across the Arthropoda: Origin and evolutionary history of the chemosensory system. Genome Biol. Evol. 3, 476-490 (2011).

Page $11 / 27$ 
45. Steidle, J. L. M. \& Van Loon, J. J. A. Chemoecology of parasitoid and predator oviposition behavior (ed. Hilker M. \& Meiners T.) 291-317 (2002).

46. Zheng, Y. N., Qi, J. Y., Sun, S. H. \& Yang, C.C. Advance in research of Chouioia cunea Yang (Hymenoptera: Eulophidade) and its biocontrol application in China. Chinese J. Biol. Control. 28, 275-281 (2012).

47. Yuan, X., Fan, H. L. \& Li, D. S. Effects of insect-resistant transgenic rices on enemies in paddy fields: a review. Guangdong Agri. Sci. 44, 59-65 (2017).

48. Ozaki, M. et al. Ant nestmate and nonnestmate discrimination by a chemosensory sensillum. Sci. 309, 311-314 (2005).

49. Zhang, S., Zhang, Y. J., Su, H. H., Gao, X. W. \& Guo Y. Y. Binding characterization of chemosensory protein MmedCSP1 in Microplitis mediator (Hymenoptera: Braconidae). Acta Entomol. Sin. 52, 838-844 (2009).

50. Shivaraju, C. Bio-intensive management of invasive eucalyptus gall wasp Leptocybe invasa Fisher \& La Salle (Eulophidae: Hymenoptera). PhD dissertation, University of Agricultural Sciences, Bengaluru, Kathalagere, India. Preprint at http://krishikosh.egranth.ac.in/handle/1/66048 (2012).

51. Hill, C. A. G protein-coupled receptors in Anopheles gambiae. Sci. 298, 176-178 (2002).

52. Jiao, Y. C., Moon, S. J. \& Montell, C. A Drosophila gustatory receptor required for the responses to sucrose, glucose, and maltose identified by mRNA tagging. P. Natl. A. Sci. 104, 14110-14115 (2007).

53. Scott, K. et al. A chemosensory gene family encoding candidate gustatory and olfactory receptors in Drosophila. Cell 104, 661-673 (2001).

54. Ebbs, M. L. \& Amrein, H. Taste and pheromone perception in the fruit fly Drosophila melanogaster. Pflugers Arch. 454, 735-747 (2007).

55. Kent, L. B. \& Robertson, H. M. Evolution of the sugar receptors in insects. BMC Evol. Biol. 9, 41 (2009).

56. Feng, M. X. Study on biology and ecology of Quadrastichus mendeli Kim \& La Salle (Hymenoptera: Eulophidae). Master' thesis, University of Hainan, China. (2016). (in Chinese with English abstract)

57. Robertson, H. M., Gadau, J. \& Wanner, K. W. The insect chemoreceptor superfamily of the parasitoid jewel wasp Nasonia vitripennis. Insect Mol. Biol. 19, 121-136 (2010).

58. Blaul, B. \& Ruther, J. How parasitoid females produce sexy sons: a causal link between oviposition preference, dietary lipids and mate choice in Nasonia. Proc. R. Soc. B. 278, 3286-3293 (2011).

59. Koh, T. W. et al. The Drosophila IR20a clade of ionotropic receptors are candidate taste and pheromone receptors. Neuron $\mathbf{8 3}, 850-865$ (2014).

60. Liman, E. R., Zhang, Y. V. \& Montell, C. Peripheral coding of taste. Neuron 81, 984-1000 (2014).

61. Chen, C. et al. Drosophila ionotropic receptor 25a mediates circadian clock resetting by temperature. Nature 52, 516-520 (2015).

62. Ni, L. et al. The ionotropic receptors IR21a and IR25a mediate cool sensing in Drosophila. eLife 5, e13254 (2016).

63. Li, Z. B. et al. Identification of leg chemosensory genes and sensilla in the Apolygus lucorum. Front. Physiol. 11, 276 (2020).

64. Greppi, C. et al. Mosquito heat seeking is driven by an ancestral cooling receptor. Sci. 367, 681-684 (2020).

65. Gong, D. P., Zhang, H. J., Zhao, P., Xia, Q. Y. \& Xiang, Z. H. The odorant binding protein gene family from the genome of silkworm, Bombyx mori. BMC Genomics 10, 332 (2009).

66. Hekmat-Scafe, D. S. Genome-wide analysis of the odorant-binding protein gene family in Drosophila melanogaster. Genome Res. 12, 1357-1369 (2002).

67. Richards, S. et al. The genome of the model beetle and pest Tribolium castaneum. Nature 452, 949-955 (2008).

68. Xu, P. X., Zwiebel, L. J. \& Smith, D. P. Identification of a distinct family of genes encoding atypical odorant-binding proteins in the malaria vector mosquito, Anopheles gambiae. Insect Molec. Biol. 12, 549-560 (2003).

69. Zhou, Y. et al. Expression and functional characterization of odorant binding protein genes in the endoparasitic wasp Cotesia vestalis. Insect Sci. 0, 1-15 (2020).

70. Li, K. et al. Odorant binding characteristics of three recombinant odorant binding proteins in Microplitis mediator (Hymenoptera: Braconidae). J. Chem. Ecol. 40, 541-548 (2014).

71. Li, K. M., Ren, L. Y., Zhang, Y. J., Wu, K. M. \& Guo, Y. Y. Knockdown of Microplitis mediator odorant receptor involved in the sensitive detection of two chemicals. J. Chem. Ecol. 38, 287-294 (2012).

72. Jones, W. D., Nguyen, T. A., Kloss, B., Lee, K. J. \& Vosshall, L. B. Functional conservation of an insect odorant receptor gene across 250 million years of evolution. Curr. Biol. 15, 119-121 (2005).

73. Swarup, S., Williams, T. I. \& Anholt, R. R. H. Functional dissection of odorant binding protein genes in Drosophila melanogaster. Genes Brain Behav. 10, 648-657 (2011).

74. Crava, C. M., Sassù, F., Tait, G., Becher, P. G. \& Anfora, G. Functional transcriptome analyses of Drosophila suzukii antennae reveal mating-dependent olfaction plasticity in females. Insect Biochem. Mol. Biol. 105, 51-59 (2019).

75. Gu, S. H. et al. Molecular identification and differential expression of sensory neuron membrane proteins in the antennae of the black cutworm moth Agrotis ipsilon. J. Insect Physiol. 59, 430-443 (2013).

76. Halty-deLeon, L., Miazzi, F., Kaltofen, S., Hansson, B. S. \& Wicher, D. The mouse receptor transporting protein RTP1S and the fly SNMP1 support the functional expression of the Drosophila odorant coreceptor Orco in mammalian culture cells. J. Neurosci. Methods. 271, 149-153 (2016).

77. Shan, S. et al. Molecular characterization and expression of sensory neuron membrane proteins SNMPs in the parasitoid Microplitis mediator (Hymenoptera: Braconidae). Insect Sci. 27,425-439 (2019).

78. Rytz, R., Croset, V. \& Benton, R. Ionotropic receptors (IRs): Chemosensory ionotropic glutamate receptors in Drosophila and beyond. Insect Biochem. Molec. 43, 888-897 (2013). 
79. Getahun, M. N., Wicher, D., Hansson, B. S. and Olsson, S. B. Temporal response dynamics of Drosophila olfactory sensory neurons depends on receptor type and response polarity. Front. Cell. Neurosci. 6 (2012).

\section{Figures}

(a)

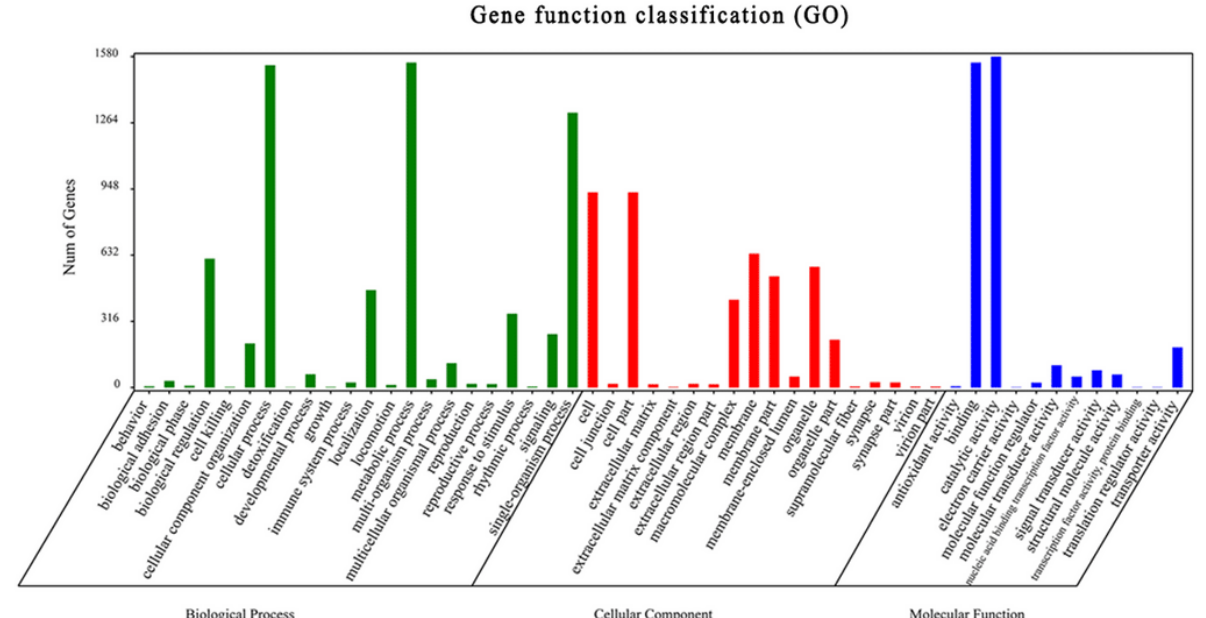

(b)

Biological Process

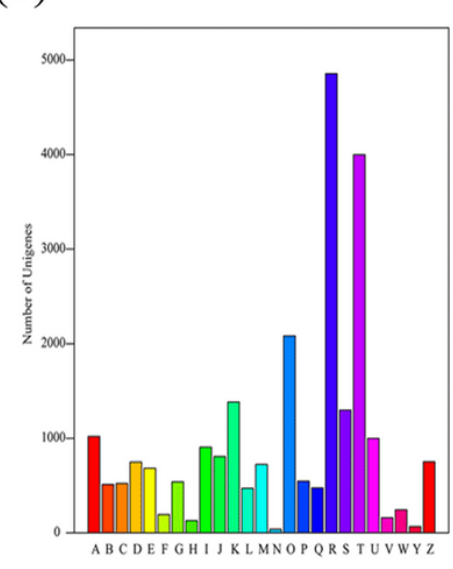

Function Class (c)

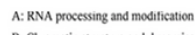

B: Chromatin structure and dynamies

C: Encregy production and conversion

D: Cell cycle control, ell division, chromosome partitioning

E: Amino acid transport and metabolism

F: Nucleotide transport and metabolism

G: Carbohydrate transport and metabolism

H. Connzyme transport and metaboll

J: Translation, ribosomal structure and biogenesis

K: Transcription

L: Replication, recombination and repair

N: Cell motility

O: Postrtranslational modification, protcin turnover, chaperon

P: Inorganic ion transport and metabolism

Q: Secondary metabolites biosynthesis, transport and catabo

R: General function prediction only

S: Function unknown

Thansuction mechanisms

W

$V:$ Defense mechanisms

Y: Nuclear structure

Z: Cyoskcleton
KEGG classification

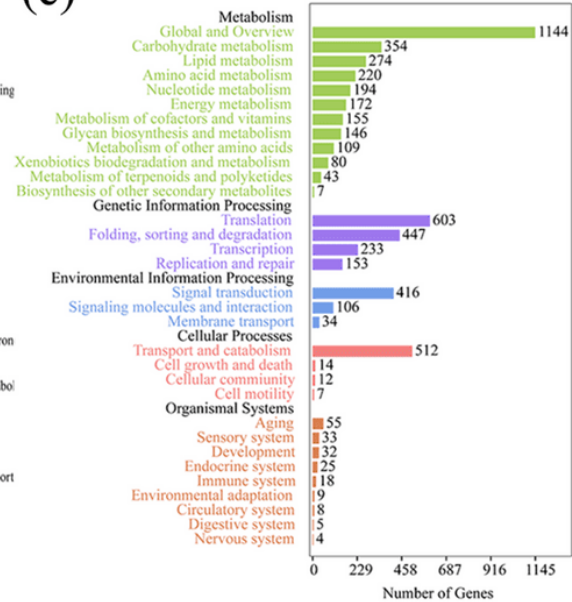

Figure 1

Distribution of transcriptome contigs from Quadrastichus mendeli adults. (a) Gene Ontology (GO) analysis. (b) Eukaryotic Orthologous Groups of protein (KOG) classification. (c) Kyoto Encyclopedia of Genes and Genomes (KEGG) classification. ( $n=15623)$. 
(a)

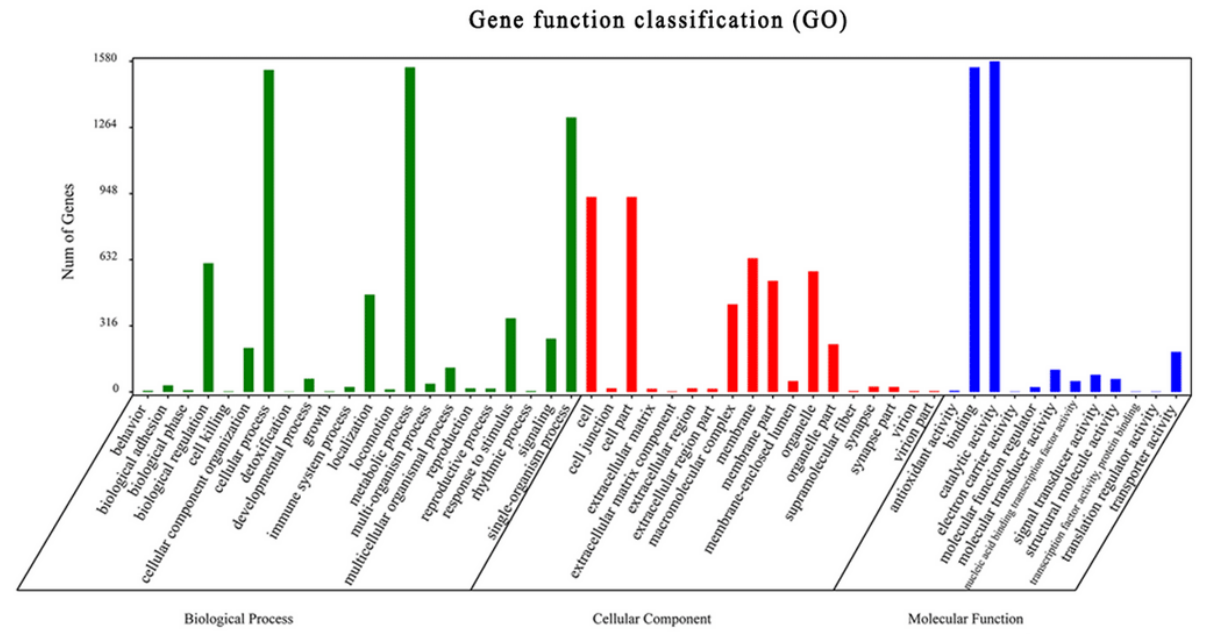

(b)

Biological Process

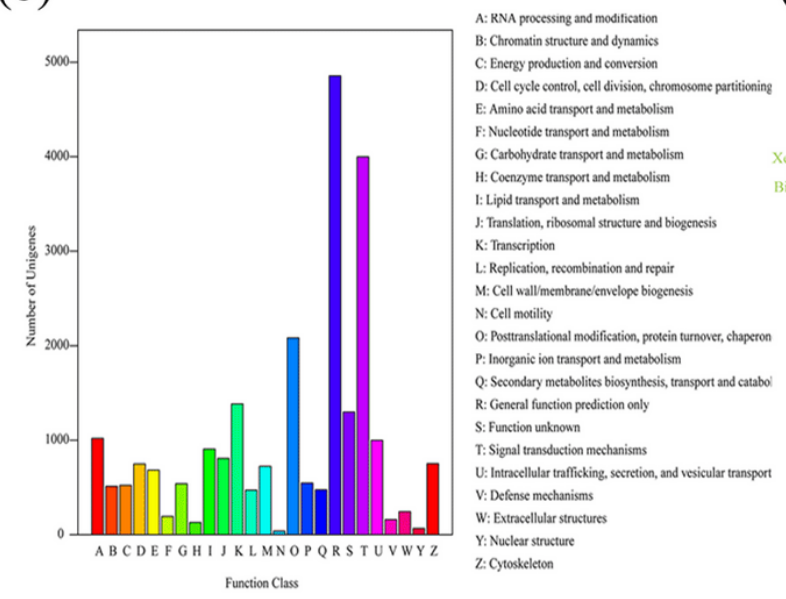

(c) KEGG classification

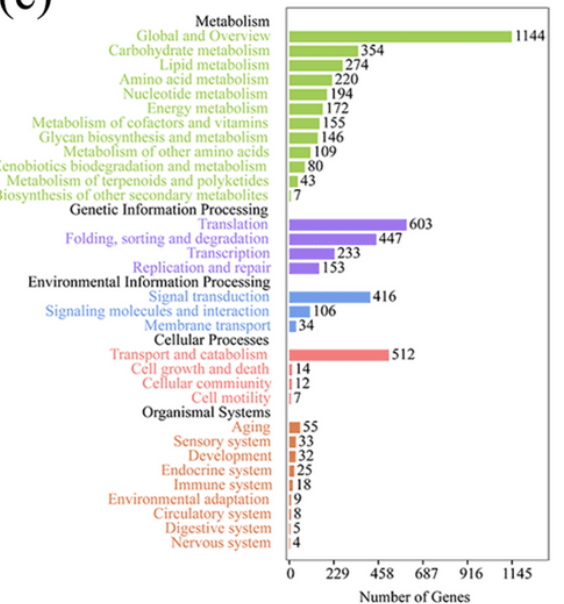

\section{Figure 1}

Distribution of transcriptome contigs from Quadrastichus mendeli adults. (a) Gene Ontology (GO) analysis. (b) Eukaryotic Orthologous Groups of protein (KOG) classification. (c) Kyoto Encyclopedia of Genes and Genomes (KEGG) classification. $(n=15623)$. 


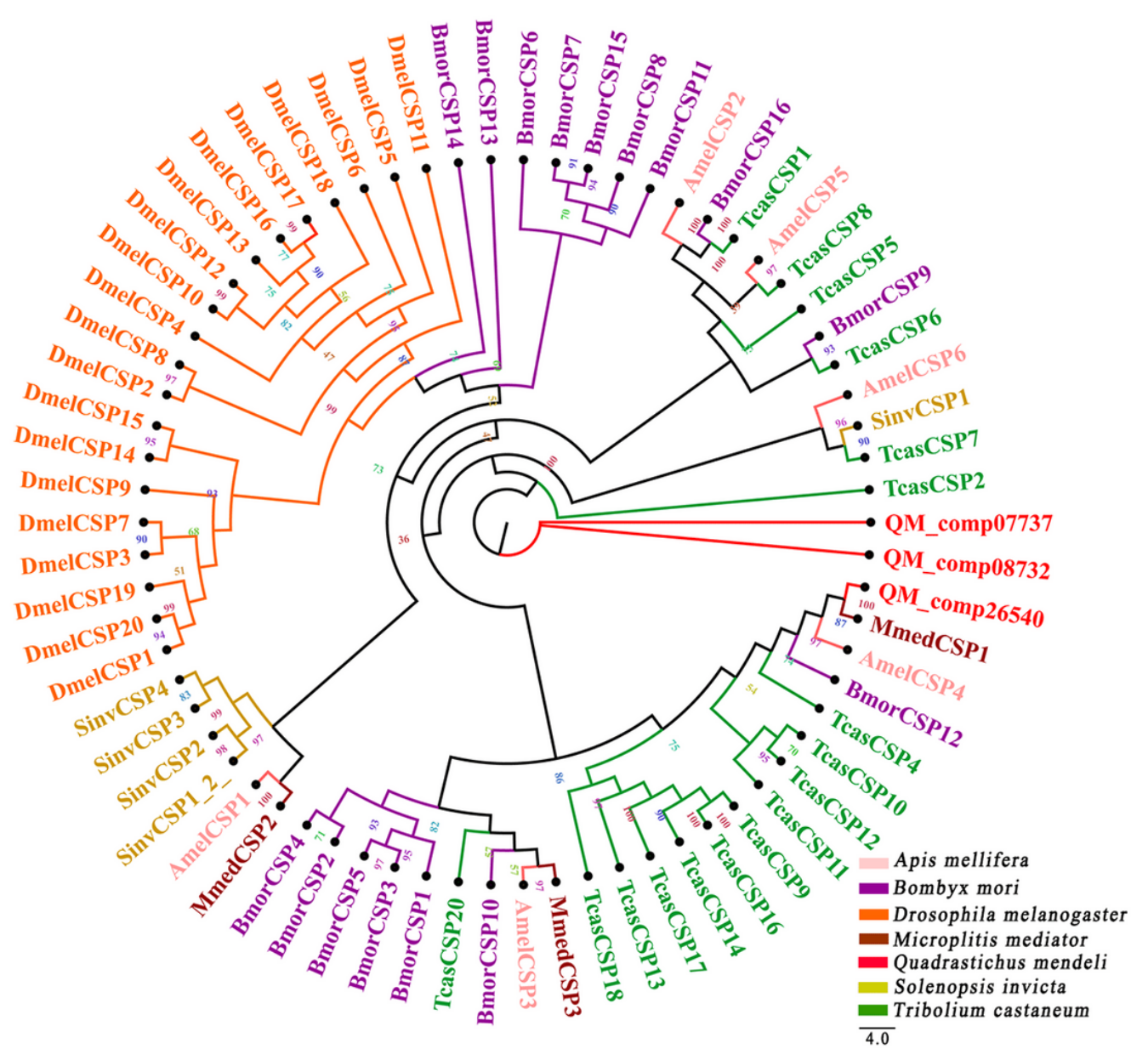

\section{Figure 2}

Phylogenetic tree of chemosensory proteins (CSPs) from Quadrastichus mendeli and other insects based on the maximum likelihood method. Included are CSPs from Apis mellifera (Amel), Bombyx mori (Bmor), Drosophila melanogaster (Dmel), Microplitis mediator (Mmed), Quadrastichus mendeli (Qmen), and Tribolium castaneum (Tcas). The specific clades are marked. Node support was assessed with 1000 bootstrap replicates. 


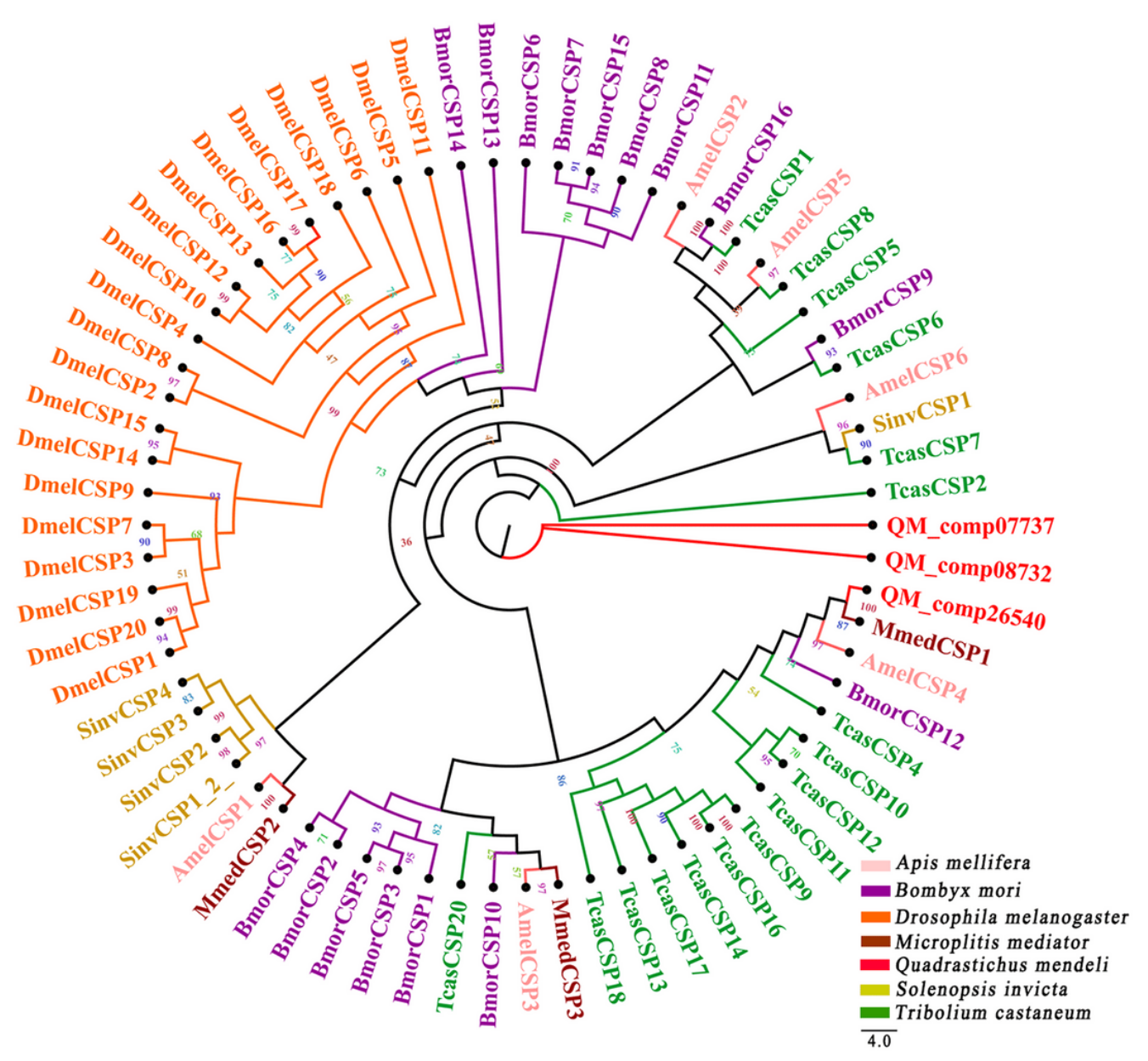

\section{Figure 2}

Phylogenetic tree of chemosensory proteins (CSPs) from Quadrastichus mendeli and other insects based on the maximum likelihood method. Included are CSPs from Apis mellifera (Amel), Bombyx mori (Bmor), Drosophila melanogaster (Dmel), Microplitis mediator (Mmed), Quadrastichus mendeli (Qmen), and Tribolium castaneum (Tcas). The specific clades are marked. Node support was assessed with 1000 bootstrap replicates. 


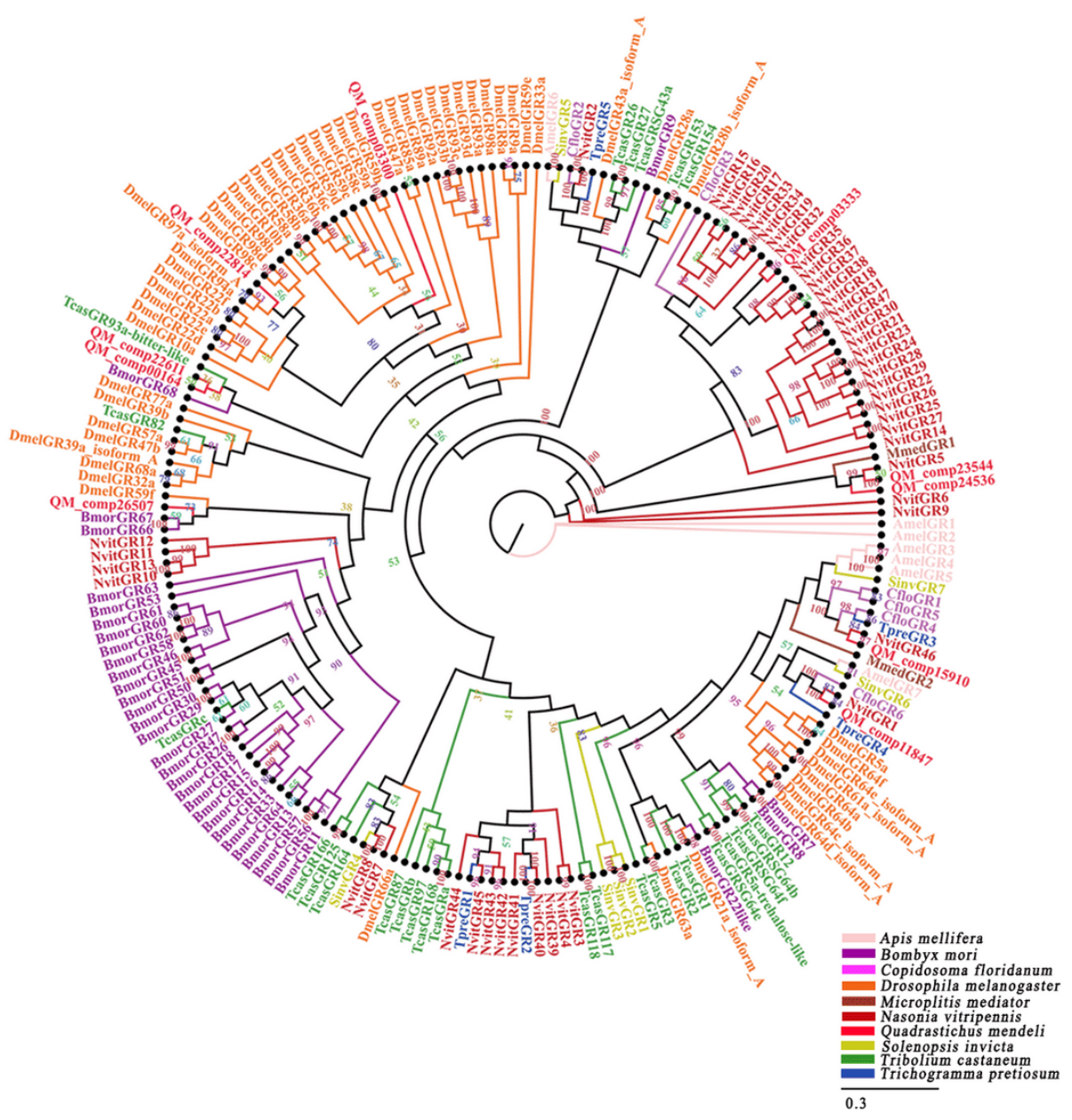

\section{Figure 3}

Phylogenetic tree of gustatory receptors (GRs) from Quadrastichus mendeli and other insects based on the maximum likelihood method. Included are OBPs from Apis mellifera (Amel), Bombyx mori (Bmor), Copidosoma floridanum (Cflo), Drosophila melanogaster (Dmel), Microplitis mediator (Mmed), Nasonia vitripennis (Nvit), Quadrastichus mendeli (Qmen), Tribolium castaneum (Tcas), and Trichogramma pretiosum (Tpre). The specific clades are marked. Node support was assessed with 1000 bootstrap replicates. 


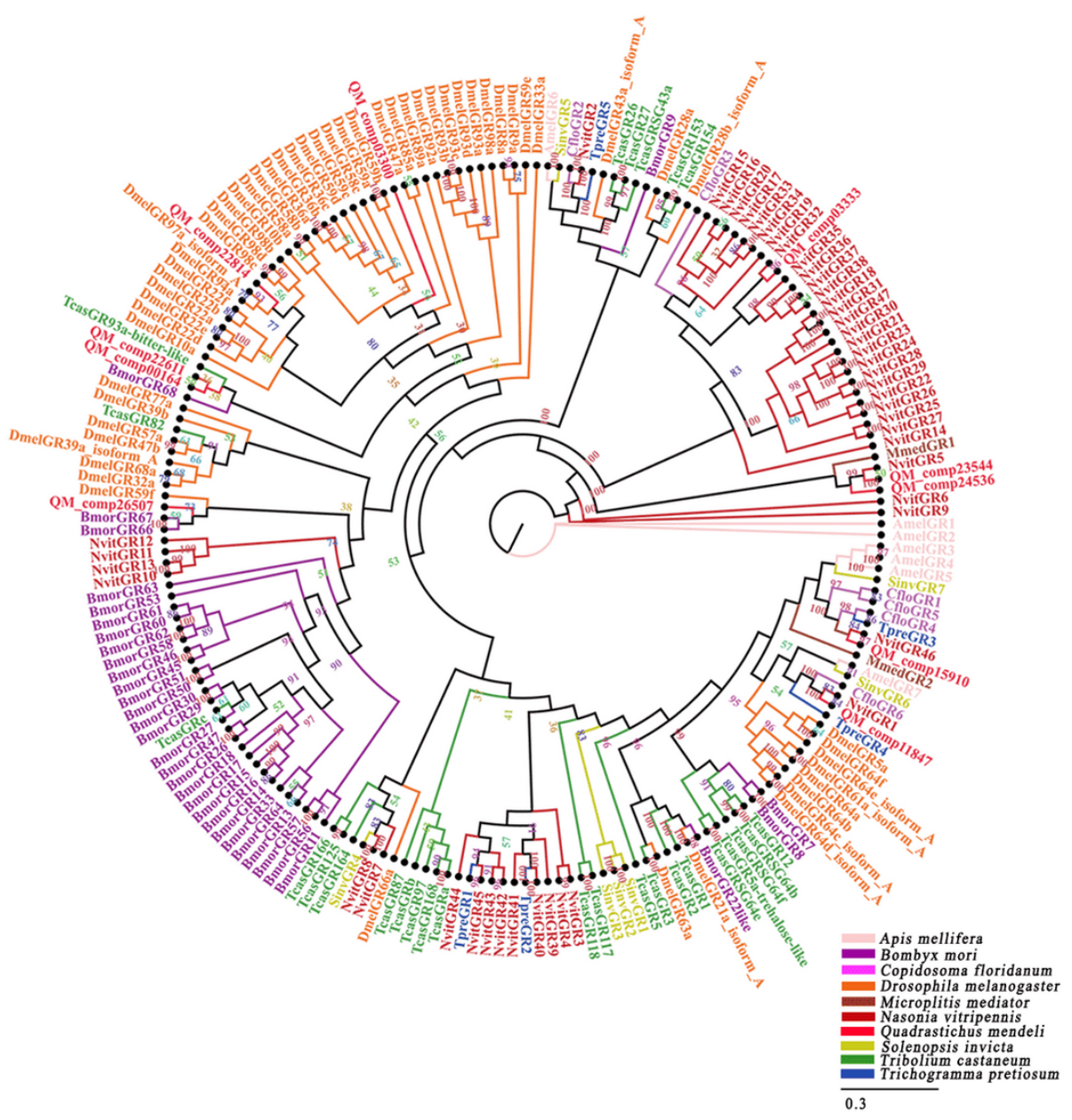

\section{Figure 3}

Phylogenetic tree of gustatory receptors (GRs) from Quadrastichus mendeli and other insects based on the maximum likelihood method. Included are OBPs from Apis mellifera (Amel), Bombyx mori (Bmor), Copidosoma floridanum (Cflo), Drosophila melanogaster (Dmel), Microplitis mediator (Mmed), Nasonia vitripennis (Nvit), Quadrastichus mendeli (Qmen), Tribolium castaneum (Tcas), and Trichogramma pretiosum (Tpre). The specific clades are marked. Node support was assessed with 1000 bootstrap replicates. 


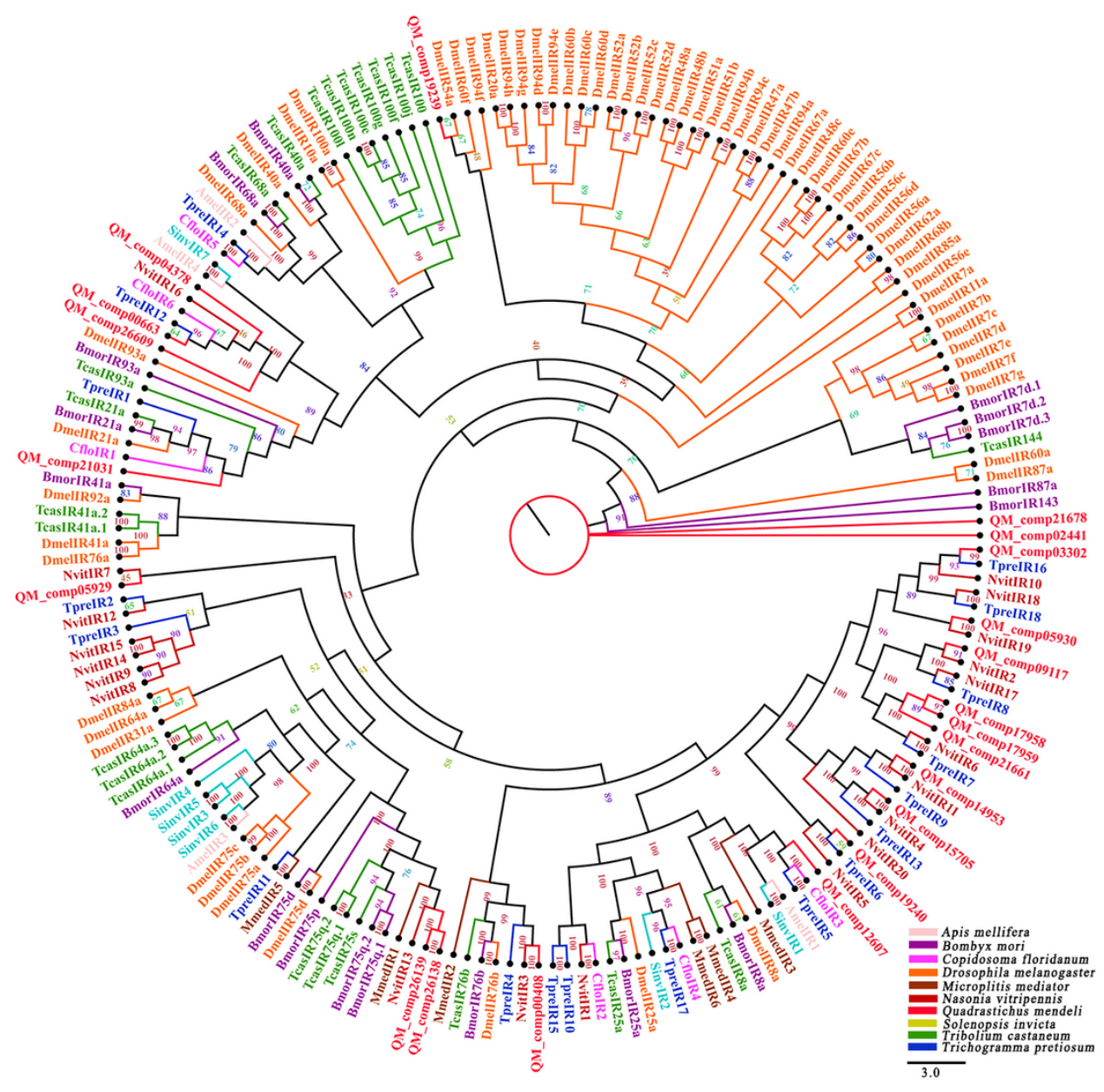

\section{Figure 4}

Phylogenetic tree of ionotropic receptors (IRs) from Quadrastichus mendeli and other insects based on the maximum likelihood method. Included are IRs from Apis mellifera (Amel), Bombyx mori (Bmor), Copidosoma floridanum (Cflo), Drosophila melanogaster (Dmel), Microplitis mediator (Mmed), Nasonia vitripennis (Nvit), Quadrastichus mendeli (Qmen), Tribolium castaneum (Tcas), and Trichogramma pretiosum (Tpre). The specific clades are marked. Node support was assessed with 1000 bootstrap replicates. 


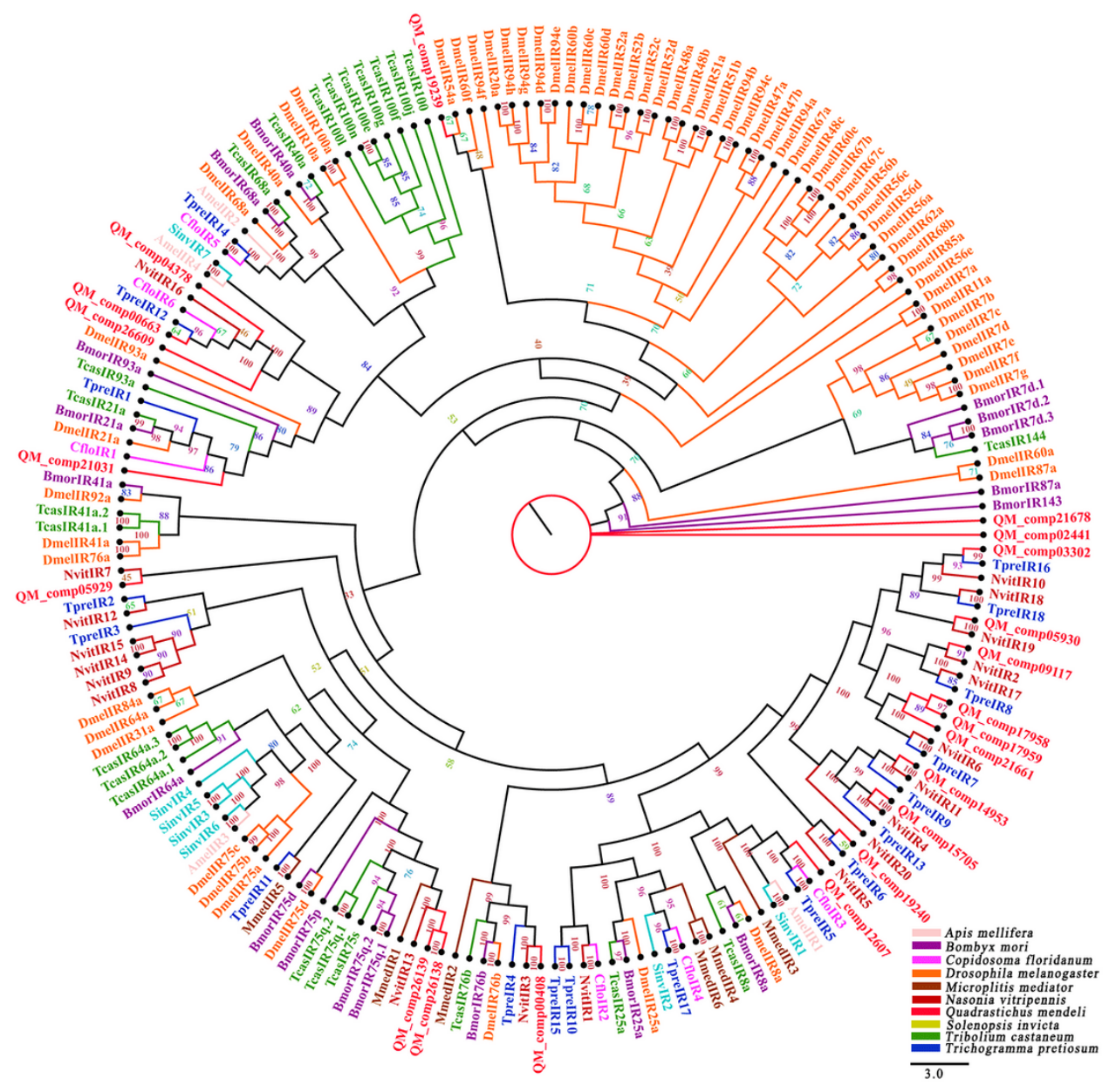

\section{Figure 4}

Phylogenetic tree of ionotropic receptors (IRs) from Quadrastichus mendeli and other insects based on the maximum likelihood method. Included are IRs from Apis mellifera (Amel), Bombyx mori (Bmor), Copidosoma floridanum (Cflo), Drosophila melanogaster (Dmel), Microplitis mediator (Mmed), Nasonia vitripennis (Nvit), Quadrastichus mendeli (Qmen), Tribolium castaneum (Tcas), and Trichogramma pretiosum (Tpre). The specific clades are marked. Node support was assessed with 1000 bootstrap replicates. 


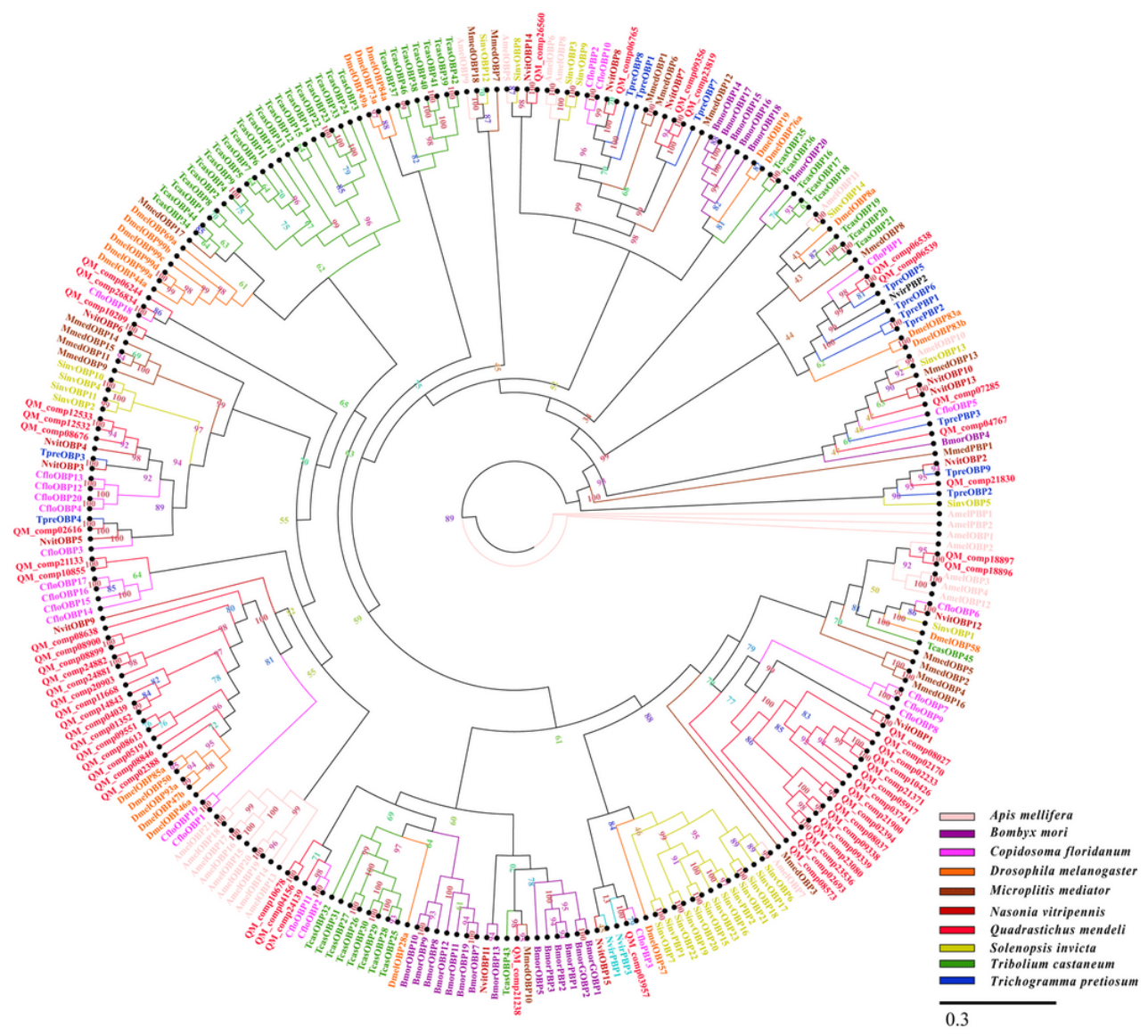

\section{Figure 5}

Phylogenetic tree of odorant binding proteins (OBPs) from Quadrastichus mendeli and other insects based on the maximum likelihood method. Included are OBPs from Apis mellifera (Amel), Bombyx mori (Bmor), Copidosoma floridanum (Cflo), Drosophila melanogaster (Dmel), Microplitis mediator (Mmed),

Nasonia vitripennis (Nvit), Quadrastichus mendeli (Qmen), Tribolium castaneum (Tcas), and Trichogramma pretiosum (Tpre). The specific clades are marked. Node support was assessed with 1000 bootstrap replicates. 


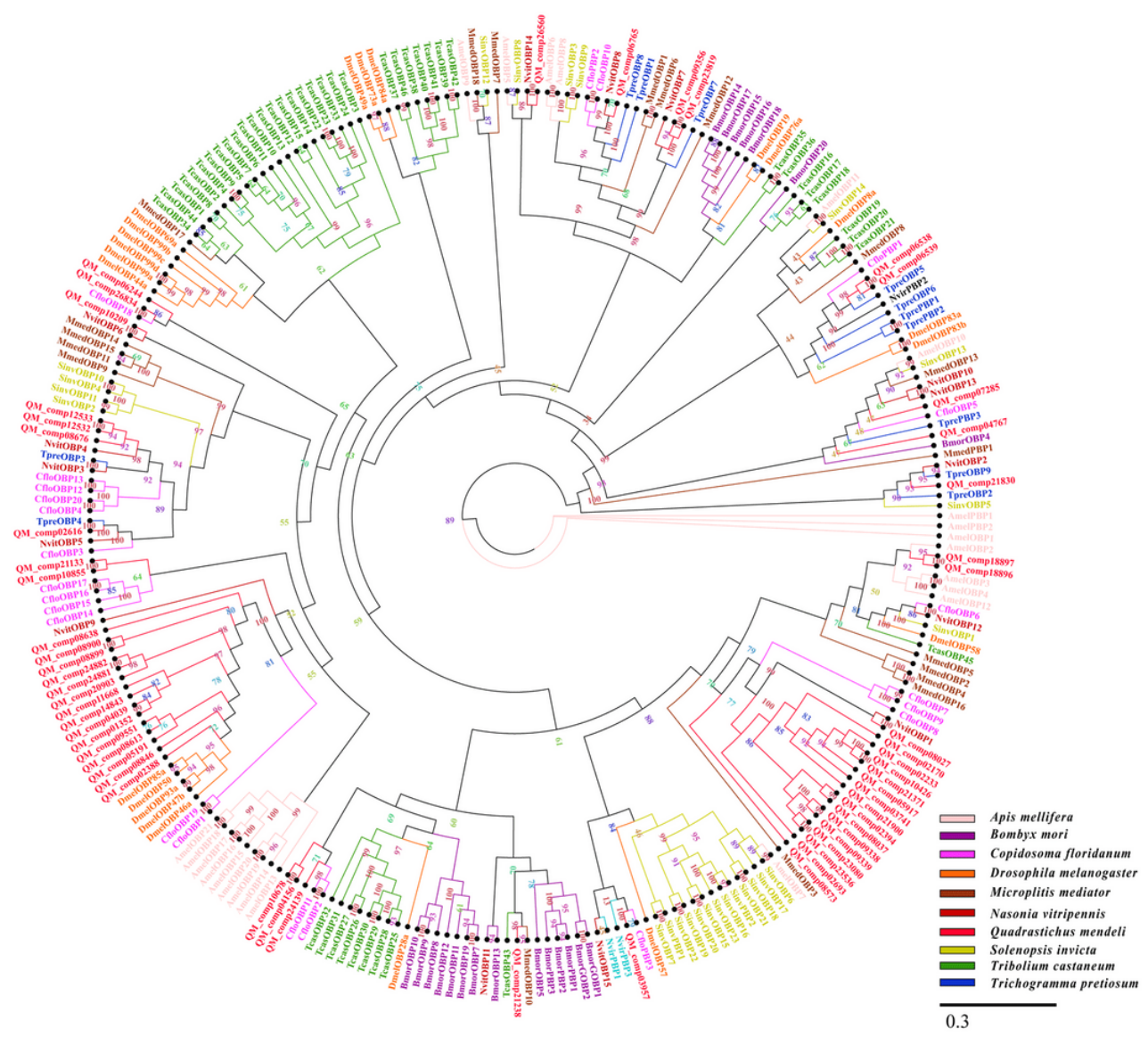

\section{Figure 5}

Phylogenetic tree of odorant binding proteins (OBPs) from Quadrastichus mendeli and other insects based on the maximum likelihood method. Included are OBPs from Apis mellifera (Amel), Bombyx mori (Bmor), Copidosoma floridanum (Cflo), Drosophila melanogaster (Dmel), Microplitis mediator (Mmed),

Nasonia vitripennis (Nvit), Quadrastichus mendeli (Qmen), Tribolium castaneum (Tcas), and Trichogramma pretiosum (Tpre). The specific clades are marked. Node support was assessed with 1000 bootstrap replicates. 


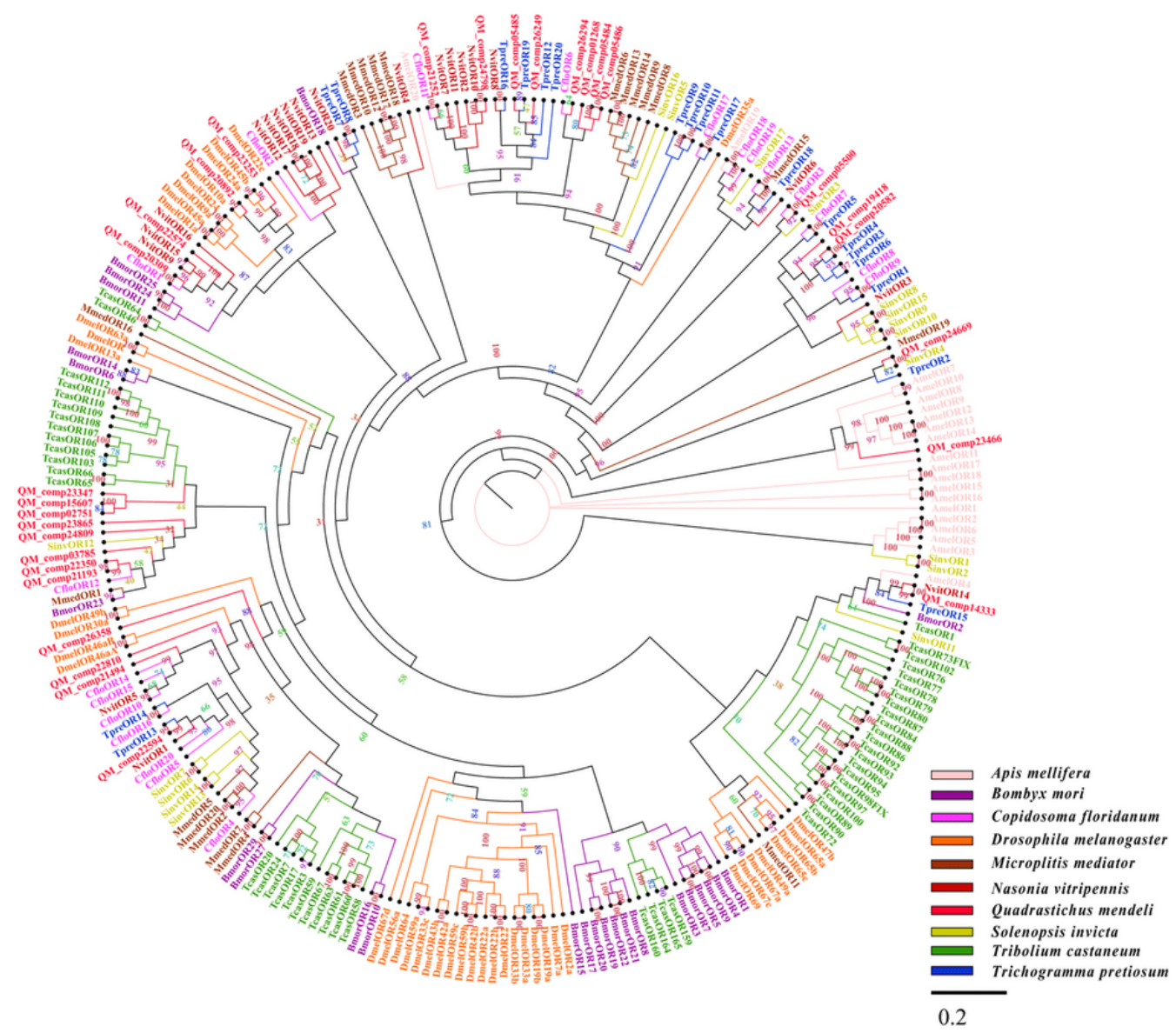

\section{Figure 6}

Phylogenetic tree of odorant receptors (ORs) from Quadrastichus mendeli and other insects based on the maximum likelihood method. Included are ORs from Apis mellifera (Amel), Bombyx mori (Bmor), Copidosoma floridanum (Cflo), Drosophila melanogaster (Dmel), Microplitis mediator (Mmed), Nasonia vitripennis (Nvit), Quadrastichus mendeli (Qmen), Tribolium castaneum (Tcas), and Trichogramma pretiosum (Tpre). The specific clades are marked. Node support was assessed with 1000 bootstrap replicates. 


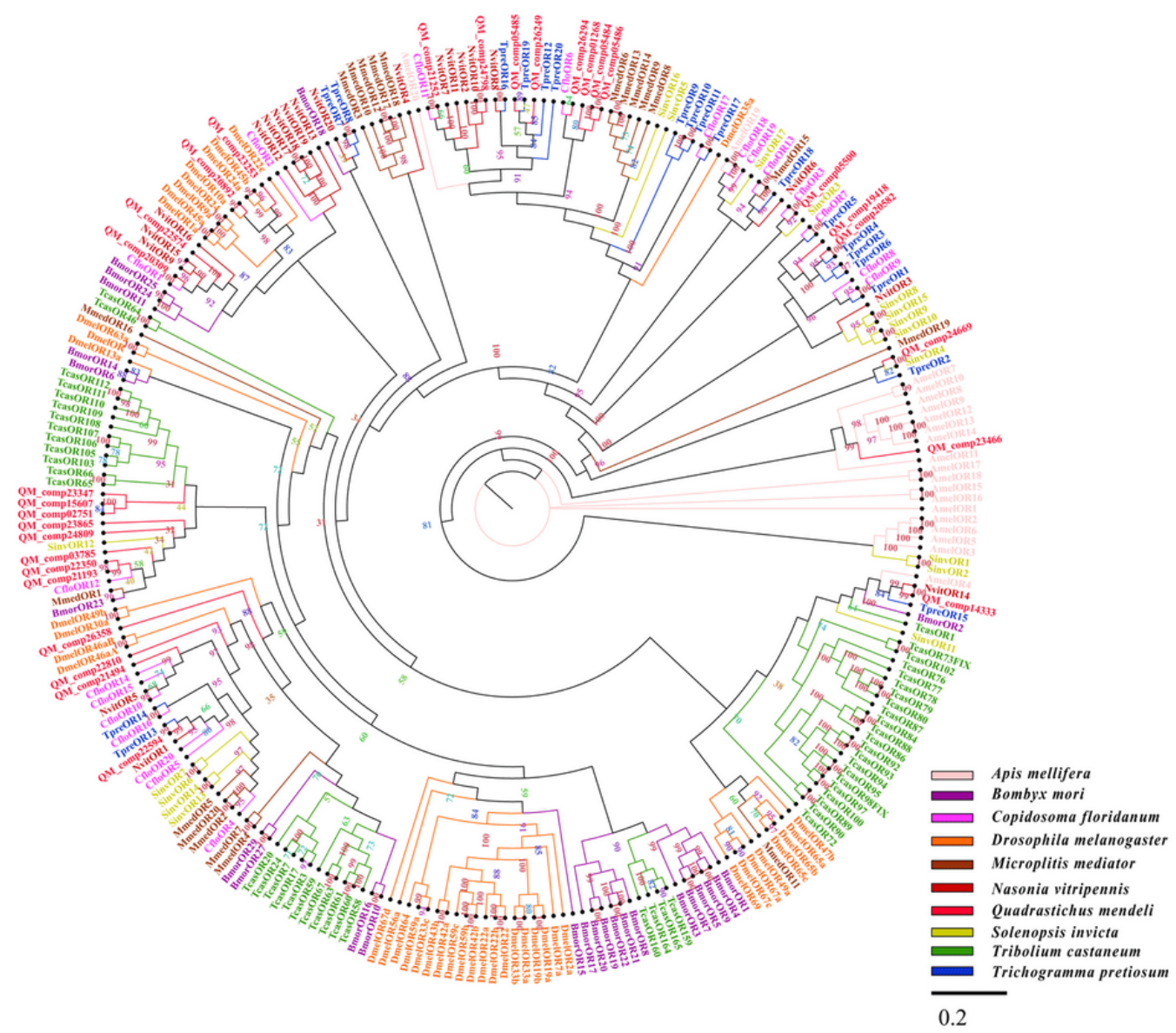

\section{Figure 6}

Phylogenetic tree of odorant receptors (ORs) from Quadrastichus mendeli and other insects based on the maximum likelihood method. Included are ORs from Apis mellifera (Amel), Bombyx mori (Bmor), Copidosoma floridanum (Cflo), Drosophila melanogaster (Dmel), Microplitis mediator (Mmed), Nasonia vitripennis (Nvit), Quadrastichus mendeli (Qmen), Tribolium castaneum (Tcas), and Trichogramma pretiosum (Tpre). The specific clades are marked. Node support was assessed with 1000 bootstrap replicates. 


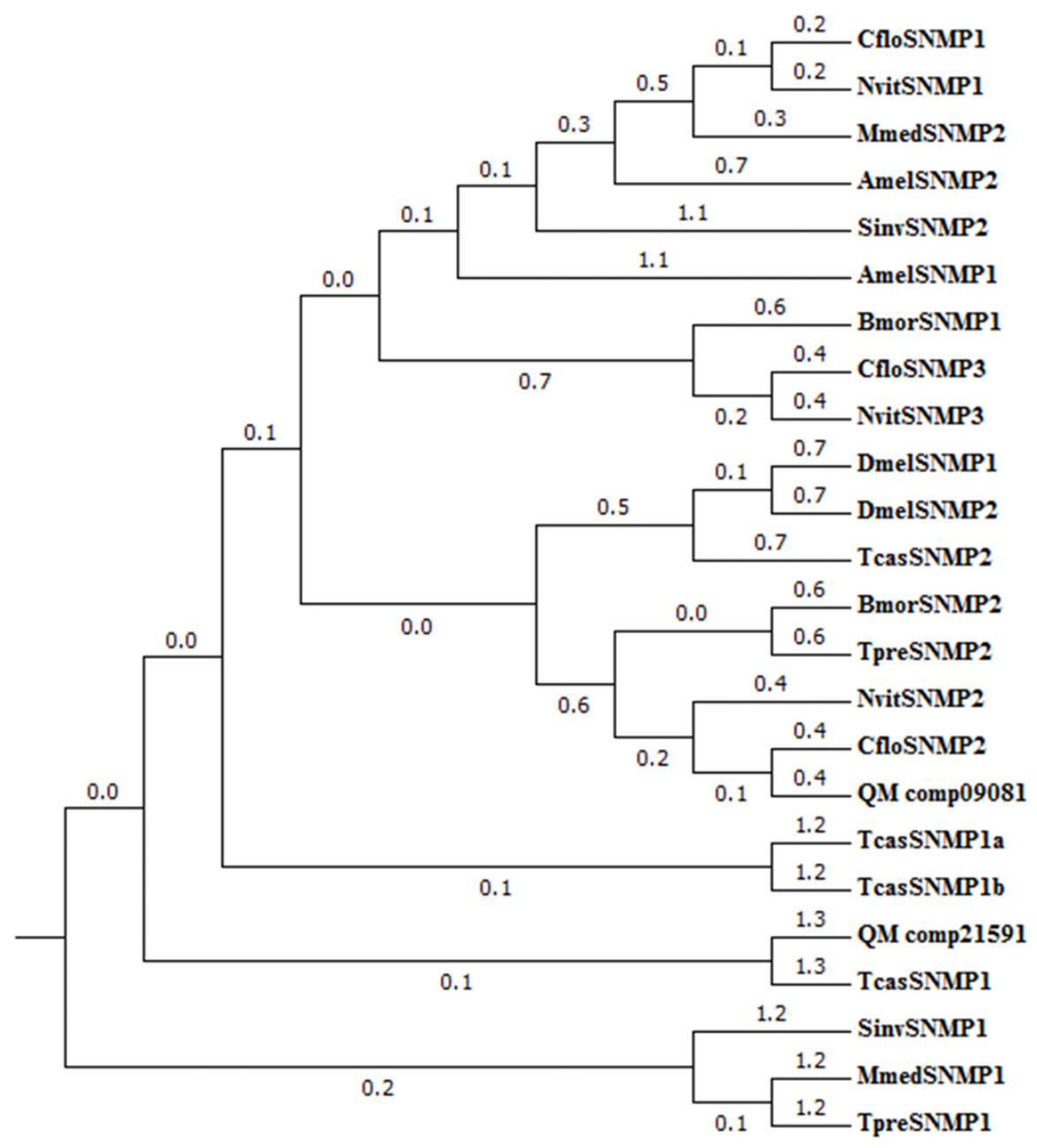

Figure 7

Phylogenetic tree of sensory neuron membrane proteins (SNMPs) from Quadrastichus mendeli and other insects based on the maximum likelihood method. Included are SNMPs from Apis mellifera (Amel), Bombyx mori (Bmor), Copidosoma floridanum (Cflo), Drosophila melanogaster (Dmel), Microplitis mediator (Mmed), Nasonia vitripennis (Nvit), Quadrastichus mendeli (Qmen), Tribolium castaneum (Tcas), and Trichogramma pretiosum (Tpre). The specific clades are marked. Node support was assessed with 1000 bootstrap replicates. 


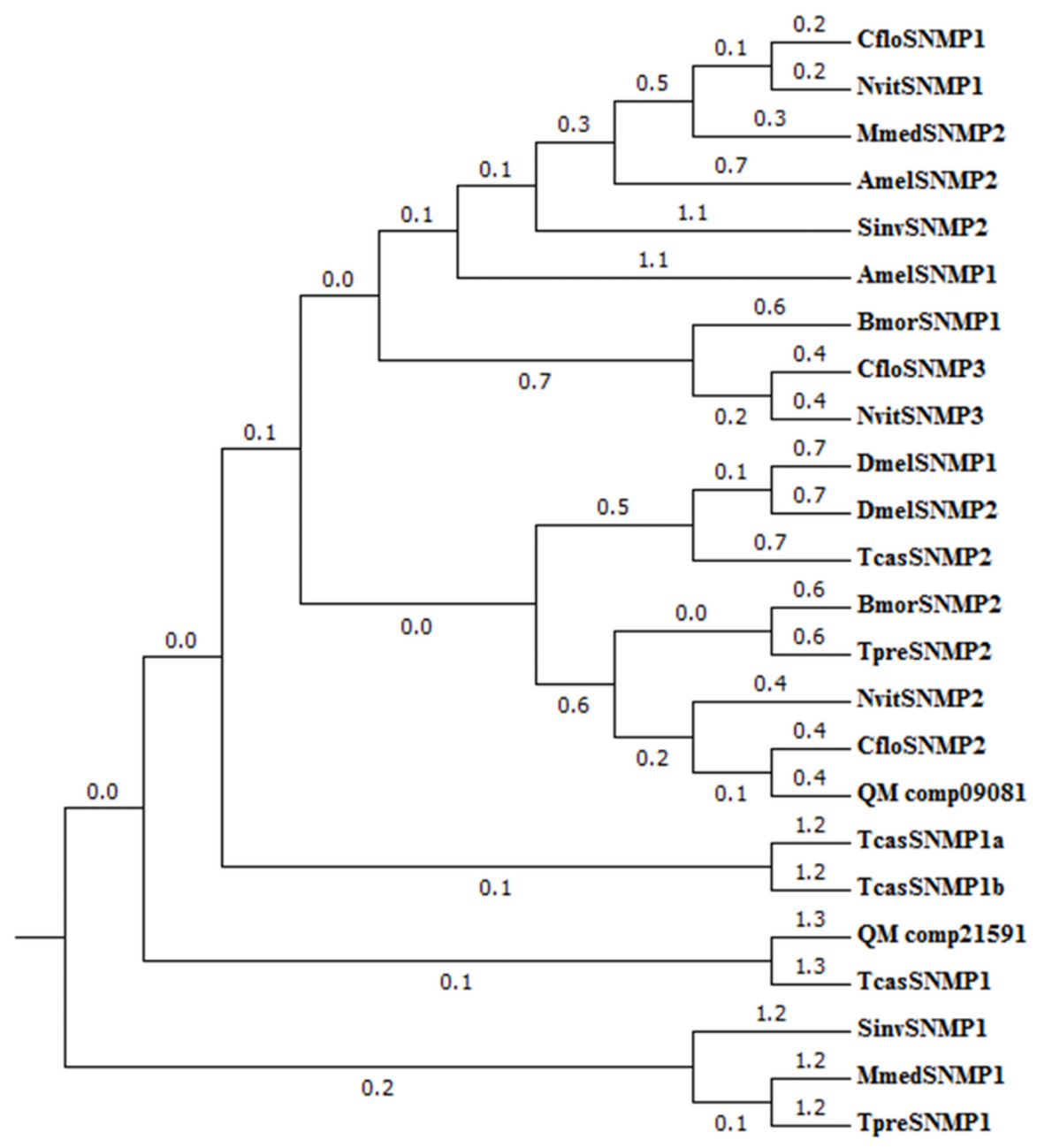

Figure 7

Phylogenetic tree of sensory neuron membrane proteins (SNMPs) from Quadrastichus mendeli and other insects based on the maximum likelihood method. Included are SNMPs from Apis mellifera (Amel), Bombyx mori (Bmor), Copidosoma floridanum (Cflo), Drosophila melanogaster (Dmel), Microplitis mediator (Mmed), Nasonia vitripennis (Nvit), Quadrastichus mendeli (Qmen), Tribolium castaneum (Tcas), and Trichogramma pretiosum (Tpre). The specific clades are marked. Node support was assessed with 1000 bootstrap replicates.

\section{Supplementary Files}

This is a list of supplementary files associated with this preprint. Click to download.

- Additionalfile9.doc

- Additionalfile9.doc

- Additionalfile8.xls

- Additionalfile8.xls

- Additionalfile7.doc

- Additionalfile7.doc

- Additionalfile6.xls

- Additionalfile6.xls

- Additionalfile5.xls

- Additionalfile5.xls

- Additionalfile4.xls

- Additionalfile4.xls

- Additionalfile3.xls

- Additionalfile3.xls 
- Additionalfile2.gif

- Additionalfile2.gif

- Additionalfile1.xls

- Additionalfile1.xls

Page 27/27 